\title{
Reactive oxygen species mediate inflammatory cytokine release and EGFR-dependent mucin secretion in airway epithelial cells exposed to Pseudomonas pyocyanin
}

\author{
B Rada ${ }^{1}$, P Gardina $^{2}$, TG Myers ${ }^{2}$ and TL Leto ${ }^{1}$
}

Despite the long-appreciated in vivo role of the redox-active virulence factor pyocyanin in Pseudomonas airway infections and the importance of airway epithelial cells in combating bacterial pathogens, little is known about pyocyanin's effect on airway epithelial cells. We find that exposure of bronchiolar epithelial cells to pyocyanin results in MUC2/MUC5AC induction and mucin secretion through release of inflammatory cytokines and growth factors (interleukin (IL)-1 $\beta$, IL-6, heparin-bound epidermal growth factor, tissue growth factor- $\alpha$, tumor necrosis factor- $\alpha$ ) that activate the epidermal growth factor receptor pathway. These changes are mediated by reactive oxygen species produced by pyocyanin. Microarray analysis identified 286 pyocyanin-induced genes in airway epithelial cells, including many inflammatory mediators elevated in cystic fibrosis (granulocyte colony-stimulating factor (G-CSF), granulocyte-monocyte CSF, chemokine (C-X-C motif) ligand 1 (CXCL1), serum amyloid, IL-23) and several novel pyocyanin-responsive genes of potential importance in the infection process (IL-24, CXCL2, CXCL3, CCL20, CXCR4). This comprehensive study uncovers numerous details of pyocyanin's proinflammatory action and establishes airway epithelial cells as key responders to this microbial toxin.

\section{INTRODUCTION}

Pseudomonas aeruginosa is an opportunistic pathogen that rarely infects healthy individuals but causes infections in immunocompromised or mechanically ventilated patients or in patients with malignancies. P. aeruginosa infects the lungs of patients with chronic obstructive pulmonary disease, chronic bronchiectasis, and cystic fibrosis (CF). ${ }^{1-3}$ In total, $80 \%$ of CF patients are colonized with $P$. aeruginosa. ${ }^{4}$ The pathogenicity of $P$. aeruginosa is due to its wide range of virulence factors. ${ }^{5}$ One of the major virulence factors is the redox-active toxin, pyocyanin. ${ }^{6}$ Pyocyanin is crucial for full in vivo virulence of Pseudomonas in plants, Caenorhabditis elegans, and Drosophila melanogaster. ${ }^{7-9}$ Pyocyanin is essential for acute and chronic lung infections in mice. ${ }^{10}$ Chronic administration of purified pyocyanin into mouse airways causes $\mathrm{CF}$ disease phenotype. ${ }^{11}$ Hypervirulence of $P$. aeruginosa in humans has been associated with pyocyanin overproduction. ${ }^{12}$ Most of the CF isolates of
P. aeruginosa secrete high amounts of pyocyanin in culture ${ }^{11,13}$ and concentrations up to $129.4 \mu \mathrm{m}$ have been detected in the sputum of $\mathrm{CF}$ and bronchiectasis patients. ${ }^{13}$

Tracheobronchiolar epithelial cells (TBECs) have a crucial role in initiation of the host's immune response to bacteria by detecting the first colonizers and by attracting phagocytes through cytokine and chemokine release. Healthy airways can eradicate $P$. aeruginosa, but in the lungs of CF or bronchiectasis patients, these mechanisms fail and Pseudomonas frequently establishes infection. ${ }^{2,14}$ TBECs have an important role in the host's failure to clear this opportunistic pathogen. In CF, reduced expression of the cystic fibrosis transmembrane conductance regulator anion transporter in the apical plasma membrane of TBECs leads to thicker mucus, lower airway surface liquid volume, and higher osmolarity, which enable establishment of Pseudomonas infection. ${ }^{3}$ In both acute and chronic respiratory infections Pseudomonas exists as biofilms, ${ }^{15}$ which are well

\footnotetext{
${ }^{1}$ Molecular Defenses Section, Laboratory of Host Defenses, National Institute of Allergy and Infectious Diseases, National Institutes of Health, Rockville, Maryland, USA. ${ }^{2}$ Genomic Technologies Section, Research Technologies Branch, National Institute of Allergy and Infectious Diseases, National Institutes of Health, Bethesda, Maryland, USA. Correspondence: TL Leto (tleto@niaid.nih.gov or tleto@nih.gov) 
protected from immune defenses. Pseudomonas-infected airways are characterized as a dysregulated hyperinflammatory environment, with high concentrations of inflammatory cytokines, mucus overproduction, and high numbers of infiltrated and activated neutrophils that significantly contribute to disease severity. ${ }^{3}$ On one hand, this is due to the reported hyperinflammatory state of CF bronchiolar epithelial cells, ${ }^{16}$ whereas, on the other, numerous late-phase Pseudomonas virulence factors have been implicated in contributing to maintenance of the hyperinflammatory conditions in CF airways. ${ }^{3}$

Epithelial cells are the first to be exposed to pyocyanin in Pseudomonas-infected airways. Pyocyanin is a secreted, membrane-permeable, heterocyclic compound that directly oxidizes intracellular pools of $\mathrm{NAD}(\mathrm{P}) \mathrm{H}$ and glutathione (GSH) and produces superoxide and downstream reactive oxygen species (ROS) that expose the host cell to oxidative stress. ${ }^{17}$ The redox-active nature of pyocyanin is thought to be responsible for most of its effects described on host cells. ${ }^{6,17}$ Although numerous studies established the in vivo relevance of pyocyanin in Pseudomonas airway infections, only a handful described mechanisms by which pyocyanin affects airway epithelial cells: inhibition of ciliary beat frequency and catalase expression, inactivation of $\alpha 1$ protease inhibitor, and release of interleukin (IL)-8. ${ }^{17}$ In order to better understand the role of airway epithelial cells in pyocyanin toxicity, we explored details of pyocyanin's action on TBECs to capture acute responses to pyocyanin without any influencing effects of neighboring tissues or infiltrating inflammatory cells.

Using pyocyanin at concentrations below average toxin levels detected in patients' airways ${ }^{13}(<10 \mu \mathrm{M})$, we found TBECs respond with inflammatory cytokine release, activation of epidermal growth factor receptor (EGFR) signaling, and mucin secretion. All these proinflammatory effects of pyocyanin on TBECs recapitulate many aspects of the CF lung disease phenotype and are due to intracellular ROS production. The toxin-initiated oxidative stress in TBECs also triggers robust changes in transcription of several inflammatory mediators that were reported at elevated levels in CF lungs. We describe additional pyocyanin-inducible genes whose relevance in Pseudomonas airway infections has not yet been reported. All together, our work contributes significantly to understanding of the complex inflammatory environment in Pseudomonas respiratory infections by describing several novel effects of pyocyanin on the airway epithelium.

\section{RESULTS}

\section{Pyocyanin induces mucin synthesis in bronchiolar epithelial cells}

Mucin production is a basic innate immune mechanism of the airways to remove entrapped bacteria. Mucus overproduction is a hallmark of chronic respiratory diseases including bronchiectasis and CF. ${ }^{18}$ Several factors (environmental pollutants, bacteria, viruses) can trigger mucin production. ${ }^{19}$ Although chronic administration of pyocyanin to mouse lungs over 12 weeks was shown to cause Goblet cell hyperplasia, no reports describe short-term effects of pyocyanin on mucin production in TBECs. ${ }^{11}$ Treatment of epithelial cells with pyocyanin induced mucin synthesis detected by periodic acidSchiff staining (Figure 1a). Quantitation of released mucins revealed a significant induction of mucin production by pyocyanin $(1.4 \mathrm{mg} / \mathrm{ml}$ in pyocyanin-treated cells vs. $0.5 \mathrm{mg} / \mathrm{ml}$ in untreated cells (Figure 1b)).

\section{Pyocyanin induces MUC5AC and MUC2 in bronchiolar epithelial cells}

In all, 20 mucin genes are known in the human genome, of which 12 are expressed in the airway epithelium; MUC5AC, MUC5B, and MUC2 are the major components of the airway mucus. ${ }^{18,19}$ To determine which mucins are responsible for pyocyanin-induced mucin production, we measured gene expression changes of all 12 airway-expressed mucin genes following pyocyanin exposure. MUC5AC showed the highest induction (133.7-fold) and MUC2 scored second highest (31.4-fold), in contrast to only 2.6-fold induction of MUC5B (Figure 1c). Therefore, we focused primarily on MUC5AC and secondarily on MUC2 throughout this study. Quantitative real-time PCR (Figure 1d and e), qualitative reverse transcriptase PCR (Figure 1c, gel pictures), and MUC5AC enzyme-linked immunosorbent assay (Figure 1f) showed time- and dose-dependent induction of MUC5AC and MUC2 mRNA and proteins by pyocyanin in TBECs. Secretion of MUC5AC appeared only $24 \mathrm{~h}$ after pyocyanin addition (Figure 1f). Western blot analysis of concentrated supernatants of pyocyanin-induced cells revealed increased amounts of secreted MUC5AC (band above $500 \mathrm{kD}$ ) (Figure 1g). We examined other airway epithelial cells for pyocyanin-induced mucin production. BEAS-2B, IB3-1, and 16HBE14o- cells are non-mucoid epithelial cells and pyocyanin failed to induce MUC2 and MUC5AC transcription (Figure 1h). To exclude the possibility that contaminating bacterial components in the pyocyanin preparations induce mucins as well, we compared pyocyanin preparations from different $P$. aeruginosa strains and culturing conditions. The mucin-inducing effect of pyocyanin did not depend on the source of the toxin; $10 \mu \mathrm{M}$ pyocyanin prepared from three different wild-type strains (PAO1, PA14, PA10145; 24-h cultures) induced MUC2 and MUC5AC in a source-independent manner (Figure 1i). Lipopolysaccharide, flagellum, or pilus were not responsible for mucin induction as pyocyanin preparations from $\mathrm{PAO} 1$ strains lacking each of these virulence factors induced MUC2 and MUC5AC to the same extent as that from wild-type bacteria (Figure 1i). Equivalent extracts prepared from supernatants of pyocyanin-deficient strains (PA14 PhzM, PAO1 Phz1/2, or PA 15442; 24h) or from 4-h cultures of the wild-type strains (when pyocyanin production is still absent) were without any effect (Figure 1i). These data confirm induction of MUC5AC and MUC2 by pyocyanin and not by other bacterial components.

\section{ROS mediate mucin production induced by pyocyanin}

Pyocyanin is a redox-active toxin causing oxidative stress in the host cell. ${ }^{17}$ Superoxide anions are detected in TBECs exposed to pyocyanin (Figure 2a). Superoxide anions produced in the intracellular space can dismutate to hydrogen peroxide $\left(\mathrm{H}_{2} \mathrm{O}_{2}\right)$, which can leak from cells. $\mathrm{H}_{2} \mathrm{O}_{2}$ is detected in both the 

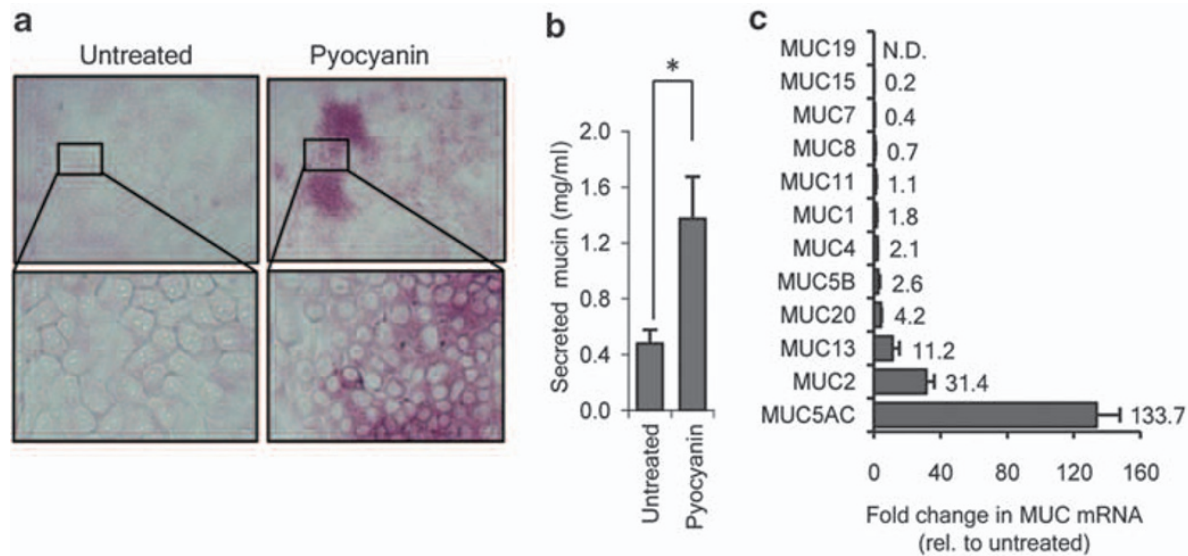

f
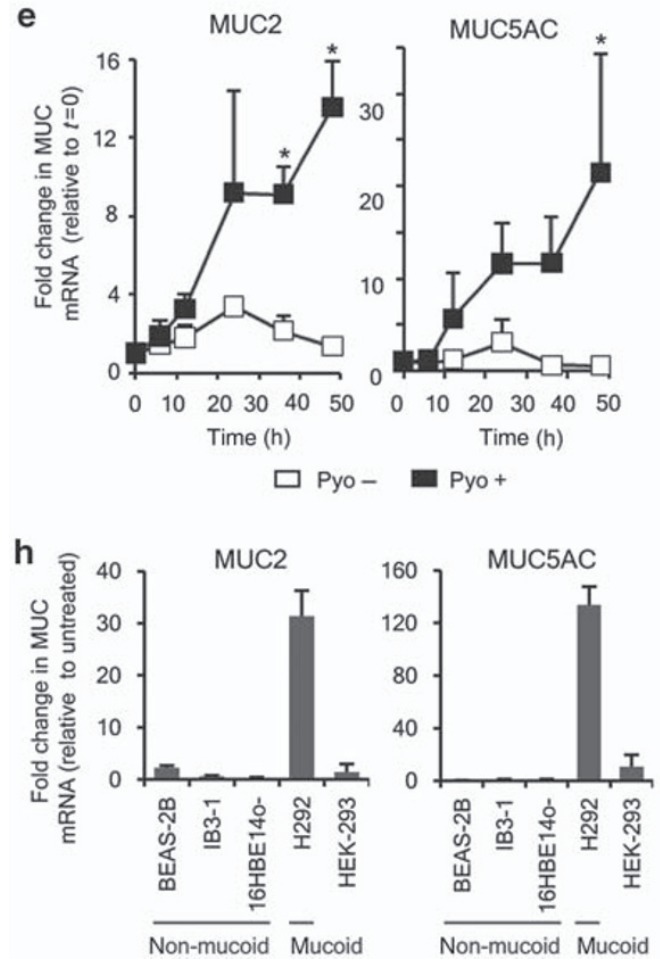

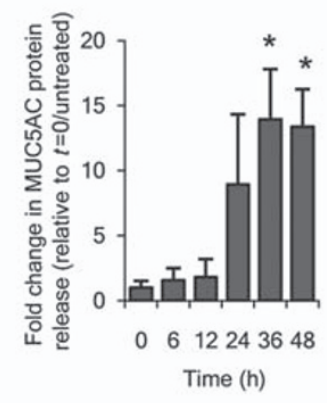

i

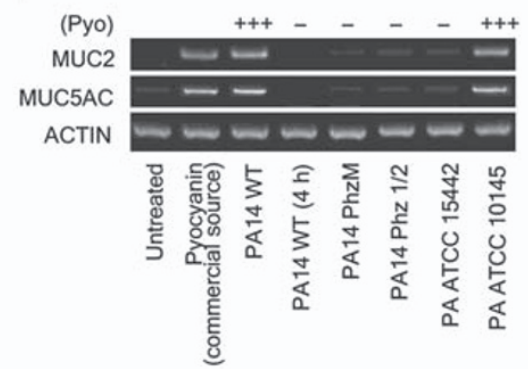

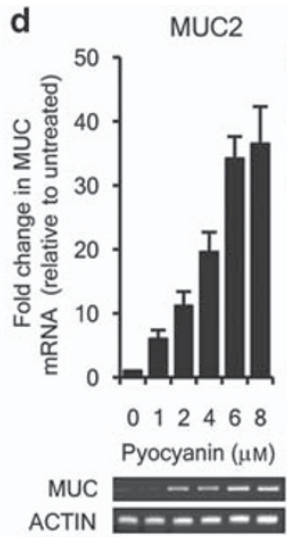

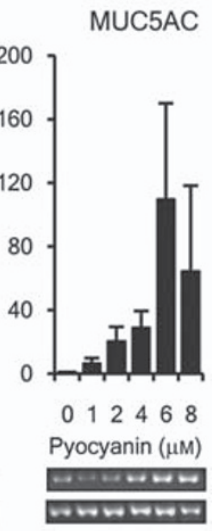

g

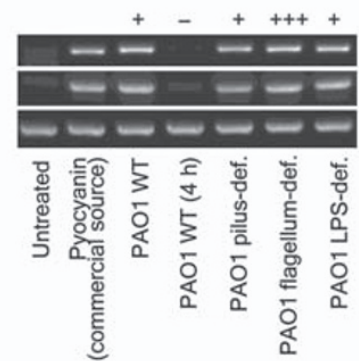

Figure 1 Pyocyanin induces mucin synthesis in bronchiolar epithelial cells. (a) Pyocyanin $(10 \mu \mathrm{M}, 2$ days) induces mucin production in bronchiolar epithelial cells as detected by periodic acid-Schiff staining (one representative result shown, $n=3$ ). (b) Quantitation of released mucins by dot-blot assay reveals significant increase in mucin production in cells exposed to pyocyanin (mean \pm s.e.m., $n=3$ ). (c) A survey of mucin transcripts expressed in the human airway epithelium identified MUC5AC and MUC2 as the two major mucins induced by pyocyanin (mean \pm s.e.m., $n=4$, quantitative (q)PCR). Pyocyanin-triggered induction of MUC5AC and MUC2 gene expression is dose- (d) and time-dependent (e) (mean \pm s.e.m. of at least three independent experiments). Primers used: graphs (qPCR): MUC2-2, MUC5AC-2; gels (reverse transcriptase PCR): MUC2-1, MUC5AC-1 (Supplementary Table S5 online). (f) Dose- and time-dependent release of MUC5AC protein in bronchiolar epithelial cells treated with pyocyanin (enzyme-linked immunosorbent assay, mean \pm s.e.m., $n=3$; dose dependence at 2 days; time dependence with $10 \mu \mathrm{M}$ Pyo). (g) Western blotting detects increased MUC5AC protein in supernatant of pyocyanin-exposed cells (another experiment gave similar results). (h) Only mucoid airway epithelial cells respond to pyocyanin by MUC5AC and MUC2 induction (qPCR, mean \pm s.e.m., $n=4$, primers: MUC2-2, MUC5AC-2). (i) MUC2 and MUC5AC induction in cells treated with pyocyanin $(8 \mu \mathrm{M}, 2$ days) extracted from 24 -h-old $P$. aeruginosa culture supernatants of different wild-type strains (PA14 WT, PA ATCC10145, PAO1 WT). Pyocyanin prepared from pilus-, flagellum-, and lipopolysaccharide-deficient strains of PAO1 also triggered MUC5AC and MUC2 induction. Equivalent extracts from pyocyanin-free supernatants of either short-term cultures of wild-type strains (PAO1 WT (4h), PA14 WT (4h)) or 24-h-old cultures of pyocyanin-deficient strains (PA14 PhzM, PA14 Phz1/2, PA ATCC 15442) failed to induce mucin expression. The \pm signs denote pyocyanin concentrations detected in each culture supernatant $(+++=20-100 \mu \mathrm{M} ;+=5-20 \mu \mathrm{M} ;-=$ none). Primers used are MUC2-1 and MUC5AC-1 (Supplementary Table S5 online). Def., deficient; ND, not detected; Pyo, pyocyanin; rel., relative; WT, wild type. ${ }^{\star} P<0.05$.

intracellular and extracellular space of pyocyanin-exposed cells (Figure 2b). As the $\mathrm{H}_{2} \mathrm{O}_{2}$ scavenger catalase does not enter the cell, intracellular $\mathrm{H}_{2} \mathrm{O}_{2}$ is resistant, whereas extracellular $\mathrm{H}_{2} \mathrm{O}_{2}$ is sensitive to catalase treatment (Figure 2b). Oxidative stress has been shown to trigger mucin production in TBECs. ${ }^{20}$ To study ROS dependence of pyocyanin-mediated mucin production, we treated epithelial cells with antioxidants ( $N$-acetyl cysteine (NAC), GSH, catalase (cat) and superoxide dismutase (SOD)) 

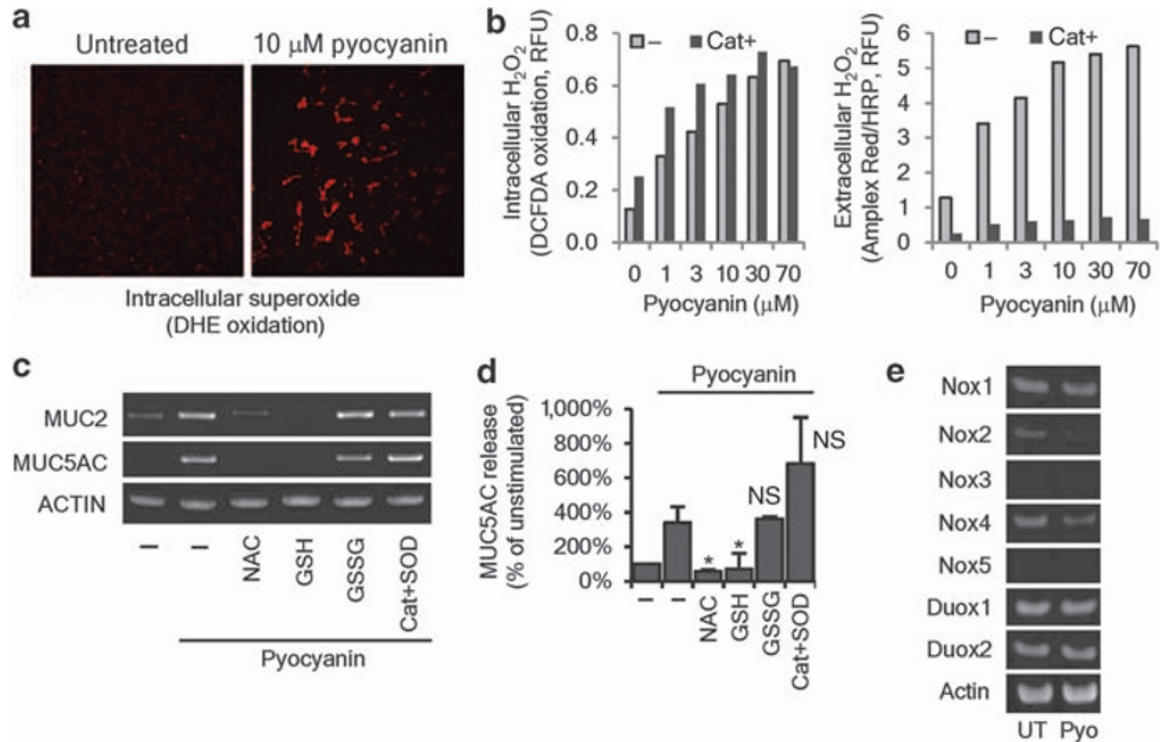

Figure 2 Reactive oxygen species (ROS) mediate pyocyanin-triggered MUC2 and MUC5AC induction. (a) Intracellular superoxide detection by dihydroethidine (DHE) oxidation in pyocyanin-treated bronchial epithelial cells ( $10 \mu \mathrm{M}$, $3 \mathrm{~h}$; one representative experiment, $n=3)$. (b) Intracellular hydrogen peroxide $\left(\mathrm{H}_{2} \mathrm{O}_{2}\right)$ detected by dichlorodihydrofluorescein diacetate (DCFDA) oxidation in cells exposed to pyocyanin (3h) is insensitive to extracellular catalase, whereas extracellular $\mathrm{H}_{2} \mathrm{O}_{2}$ release detected by Amplex Red/horseradish peroxidase (HRP) is sensitive to exogenous catalase (mean, one representative experiment, $n=3$ ). Induction of MUC2 and MUC5AC gene expression (c) and MUC5AC release (d) in pyocyanin-treated cells $(10 \mu \mathrm{M}, 2$ days) is prevented by intracellular antioxidants (10 mM N-acetyl cysteine (NAC), glutathione (GSH)). Oxidized GSH (GSSG, $10 \mathrm{mM})$ and extracellular ROS scavangers $(1500 \mathrm{U} / \mathrm{ml}$ catalase $+12.5 \mu \mathrm{g} / \mathrm{ml}$ superoxide dismutase (SOD)) have no effect (reverse transcriptase PCR: one representative experiment, $n=3$; MUC5AC release: mean \pm S.e.m., $n=4)$. (e) Pyocyanin does not increase mRNA transcripts of ROS-producing NADPH oxidases ( $10 \mu \mathrm{M}$ Pyo, 2 days, one representative, $n=4)$. NS, not significant; Pyo, pyocyanin; RFU, relative fluorescence unit. ${ }^{*} P<0.05$.

before addition of the toxin. Intracellular ROS scavengers NAC and GSH blocked MUC2 and MUC5AC induction, whereas extracellular antioxidants (cat+SOD) were without effect (Figure 2c). The oxidized form of GSH (GSSG), which has no antioxidant properties, had no effect either (Figure 2c). The same pattern of effects of these antioxidants occurs for MUC5AC release (Figure 2d). We conclude that intracellular, but not extracellular ROS, mediate pyocyanin-initiated MUC2 and MUC5AC induction.

Two members of the ROS-producing family of NADPH oxidases, Nox4 and Duox1, have been implicated in mucin synthesis in airway epithelial cells. ${ }^{21,22}$ None of the five NADPH oxidases detected were induced by pyocyanin (Figure 2e). Nox1, Duox1, and Duox 2 transcript levels remained unchanged, whereas Nox2 and Nox4 levels dropped with pyocyanin exposure (Figure 2e). Thus, induction of NADPH oxidases does not account for pyocyanin-induced ROS production.

\section{Pyocyanin activates the EGFR pathway}

Mucin production can be triggered by interactions of bacteria with TBECs, as well as by different inflammatory/immune response mediators. ${ }^{18}$ One of the major signaling pathways that mediate mucin production involves the EGFR. ${ }^{23,24}$ As oxidative stress was shown to activate EGFR and mucin secretion through MEK1/2, we tested whether a specific EGFR inhibitor (AG1478) or MEK1/2 inhibitors (U0126, PD98059) affect pyocyanin-mediated mucin induction. ${ }^{21,25}$ These inhibitors blocked induction of MUC2 and MUC5AC (Figure 3a and $\mathbf{b}$ ), whereas a platelet-derived growth factor receptor inhibitor (AG1295) or an inactive analog of AG1295 and AG1478 (AG9) had no effect (Figure 3a and $\mathbf{b}$ ), suggesting that pyocyanin induces mucin secretion entirely through EGFR.

We tested whether pyocyanin promotes proliferation of TBECs, as EGFR signaling is known to induce epithelial cell division. ${ }^{26}$ EdU (5-ethynyl-2' -deoxyuridine) labeling revealed higher numbers of actively dividing epithelial cells following pyocyanin treatment relative to untreated cultures (Figure $3 \mathbf{c}$ and $\mathbf{e}$ ), and total cell numbers were also elevated (Figure $3 \mathbf{c}$ and $\mathbf{d}$ ). Extracellular-signalregulated kinase (ERK) is a well-characterized kinase downstream from EGFR. ${ }^{26}$ We detected strong ERK phosphorylation at $24 \mathrm{~h}$ after exposure, confirming the activation of EGFR-MEK1/2-ERK pathway in pyocyanin-treated TBECs (Figure 3f).

Activation of EGFR can be ligand-dependent or -independent. ${ }^{26}$ A variety of ligands can bind and activate EGFR, whereas ROS can cause ligand-independent EGFR activation. ${ }^{26}$ To see whether pyocyanin-mediated MUC2/MUC5AC induction through EGFR requires de novo protein synthesis of potential EGFR ligands, we treated epithelial cells with the protein synthesis inhibitor cycloheximide (CHXM). CHXM completely inhibited MUC2 and MUC5AC induction by pyocyanin, suggesting that EGFR is not directly activated by oxidative stress but may act through synthesis of EGFR ligands (Figure 3a).

\section{Pyocyanin induces transcription of inflammatory cytokines and EGFR ligands}

Several inflammatory cytokines (IL- $1 \alpha,-1 \beta,-4,-5,-6,-9,-13,-17$, and tumor necrosis factor-alpha (TNF- $\alpha)$ ) and growth 


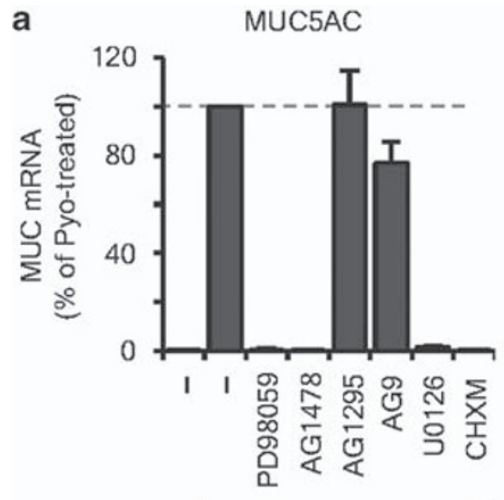

Pyocyanin

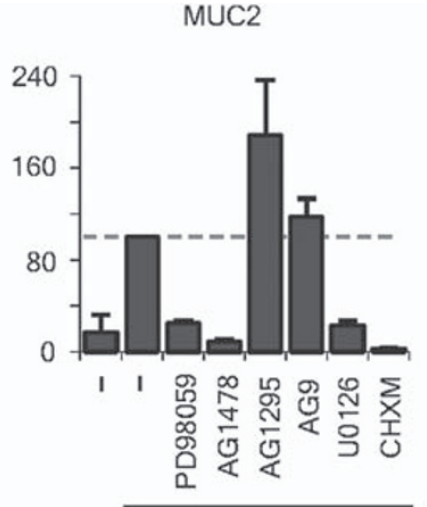

Pyocyanin

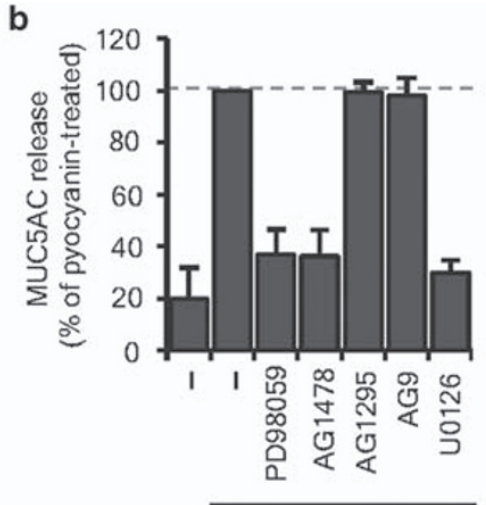

Pyocyanin

C Untreated

AG1478

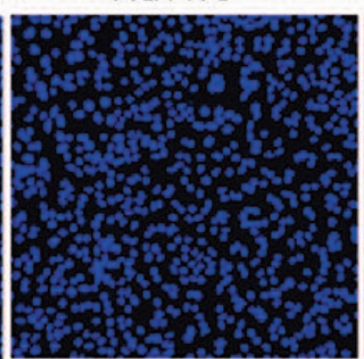

Pyocyanin
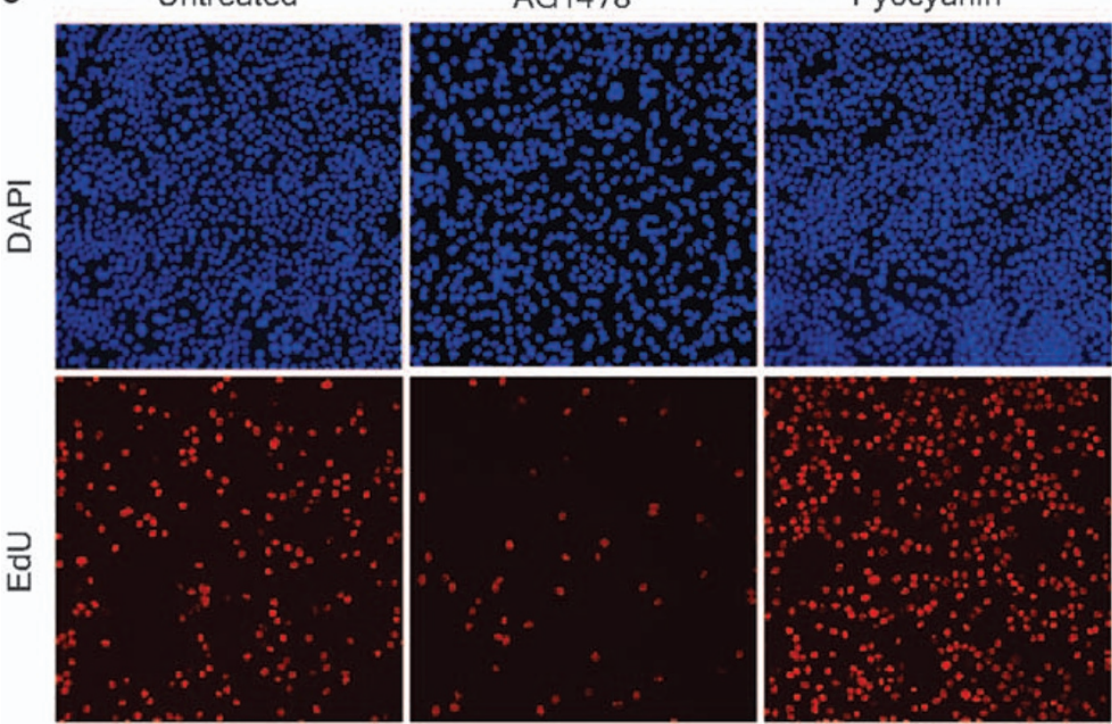

Pyocyanin+AG1478
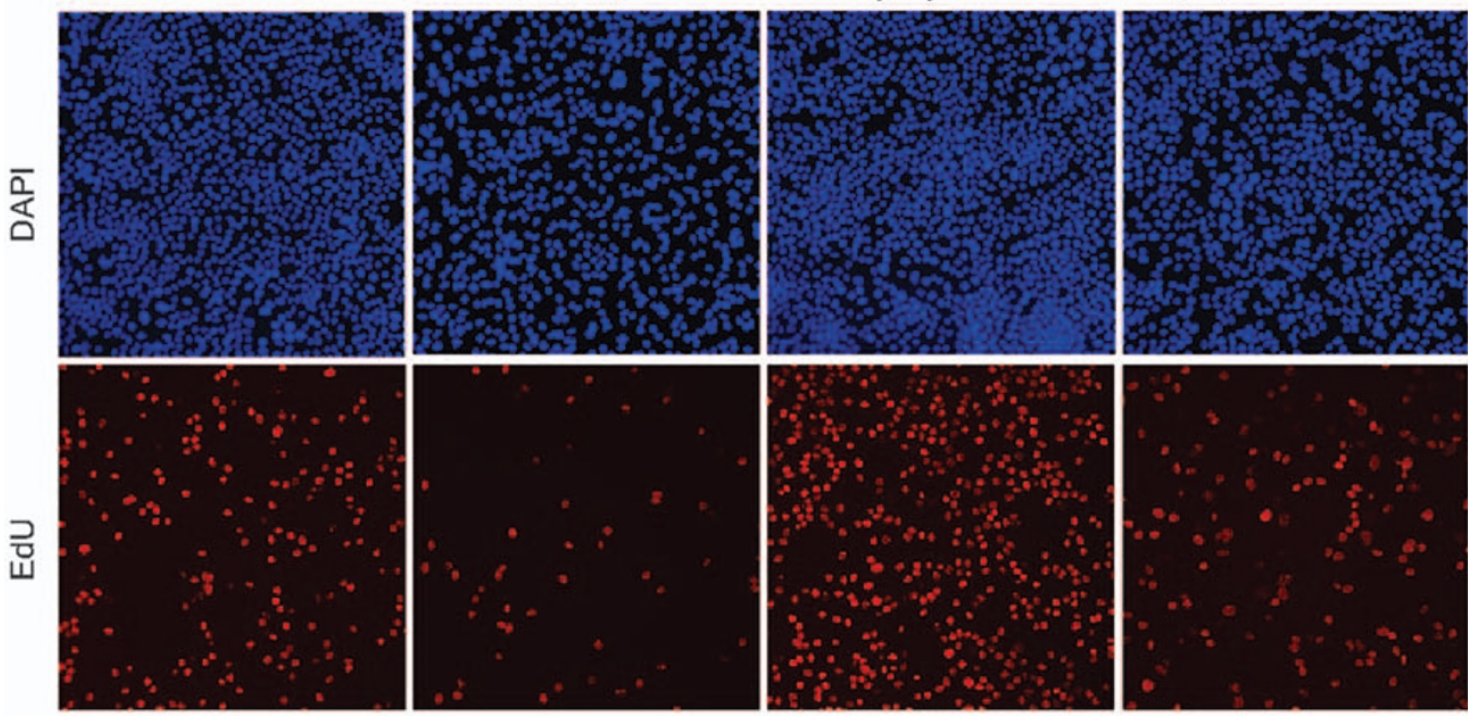

d

e

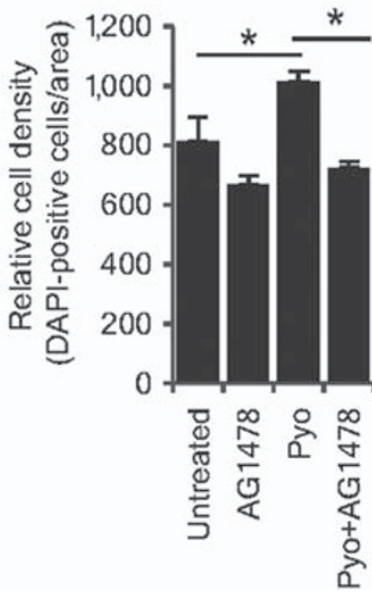

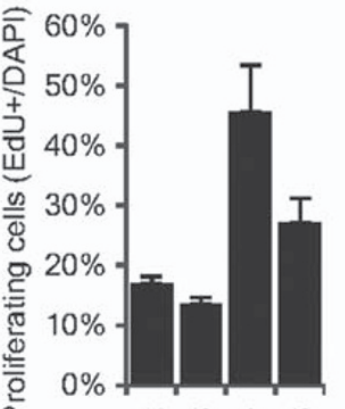

క $\infty 0 \infty$

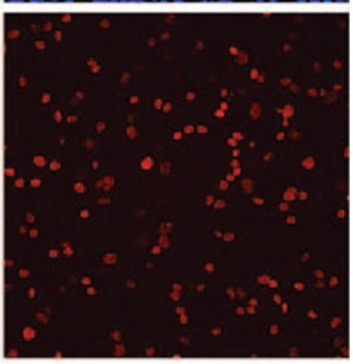

Figure 3 Pyocyanin activates epidermal growth factor receptor (EFGR) pathway and promotes proliferation in airway epithelial cells. Inhibitors of MEK1/2 (PD98059, U0126), EGFR (AG1478), and protein synthesis (cycloheximide (CHXM)) blocked pyocyanin-mediated induction of MUC2 and MUC5AC gene expression (a) and MUC5AC release (b) in bronchiolar epithelial cells. The inhibitor of the platelet-derived growth factor receptor (AG1295) or the control analog (AG9) had no effect (mean \pm S.e.m., $n=3$ ). (c) Pyocyanin-induced proliferation of bronchiolar epithelial cells is inhibited by AG1478 (detected by EdU (5-ethynyl-2' -deoxyuridine)-staining of cells treated with $8 \mu \mathrm{M}$ pyocyanin for $36 \mathrm{~h}$ ). (d) Quantitation of changes in epithelial cell density upon pyocyanin treatment (4,6-diamidino-2-phenylindole (DAPI)-stained nuclei per unit surface, mean \pm s.e.m., $n=3$ ).

(e) Pyocyanin exposure results in an increase in proportion of actively dividing epithelial cells (EdU-positive cells/all (DAPI); mean \pm s.e.m., $n=3$ ). (f) Pyocyanin greatly enhanced phosphorylation of extracellular-signal-regulated kinase (ERK) at $24 \mathrm{~h}$ after exposure in bronchiolar epithelial cells (another experiment gave similar results). t, total; $p$, phospho; Pyo, pyocyanin. ${ }^{*} P<0.05$. 
factors (EGF, heparin-bound EGF (HB-EGF), tissue growth factor-alpha (TGF- $\alpha)$ ) are known to trigger MUC2 or MUC5AC production in airway epithelial cells. ${ }^{19}$ EGF family ligands are important growth factors initiating growth and mucociliary differentiation of airway epithelial cells: amphiregulin, HB-EGF, EGF, TGF- $\alpha$, betacellulin, epigen, epiregulin, and neuregulins. ${ }^{19}$ All EGF family members are synthesized as transmembrane protein proligands present on the cell surface and are cleaved by surface proteases leading to release of the active ligand. ${ }^{27}$ A majority of the proteases responsible for this process called ectodomain shedding belong to the ADAM family of metalloproteases. ${ }^{27}$ ADAM17 (TACE) has been suggested as the major ADAM mediating MUC5AC production in TBECs. ${ }^{28}$ EGF ligands bind to ERBB family receptors (EGFR, ERBB2, ERBB3, ERBB4) and activate downstream signaling. ${ }^{26}$

Many of these genes associated with MUC5AC induction in airway epithelial cells demonstrated pyocyanin-induced transcriptional changes on human whole-genome microarrays (Figure 4a). Five cytokines (IL- $1 \alpha$, IL-1 $\beta$, IL-6, IL-8, TNF- $\alpha$ ) and two EGFR ligands (TGF- $\alpha$, HB-EGF) were strongly upregulated by pyocyanin (Figure 4a). Induction of IL- 8 by pyocyanin in TBECs was already shown. ${ }^{29}$ We also detected a 39-fold increase in IL-8 mRNA levels upon pyocyanin exposure by real-time PCR. Increased expression of IL- $1 \alpha$ ( 23 -fold), IL-1 $\beta$ (12-fold), IL-6 (391-fold), TNF- $\alpha$ (26-fold), TGF- $\alpha$ (4-fold), and HB-EGF (30-fold) was confirmed by quantitative real-time PCR (Figure $\mathbf{4 b}$ and $\mathbf{c}$ ). Significantly enhanced amounts of IL- $1 \beta$, TGF- $\alpha$, TNF- $\alpha$, and IL- 6 were detected by enzyme-linked immunosorbent assays in the supernatant of pyocyanin-treated cells compared with untreated cells (Figure 4d). Pyocyanin resulted in a 5.2-fold increase in IL-6 secretion of TBECs (Figure 4d). Pyocyanin-mediated induction of IL$1 \alpha$, IL-1 $\beta$, IL-6, TNF- $\alpha$, TGF- $\alpha$, and HB-EGF in TBECs has not been reported to date.

On the other hand, ADAM metalloproteases, MMP9, ERBB receptors, IL-17 family cytokines, or MEK1 were not induced by pyocyanin (Figure 4a, Supplementary Figure S2 online). Th2 cytokines (IL-4, -5, -9, and -13) do not mediate the effect of pyocyanin (Figure 4a). Neither increased expression (Figure 4a) nor activation of STAT6 (Supplementary Figure S1 online), the major transcription factor in IL-4/13 signaling, was elicited by pyocyanin. Pyocyanin failed to induce transcription and release of the major EGFR ligand, EGF (Supplementary Figure S2 online, Figure 4d). Thus, we identified IL- $1 \alpha$, IL-1 $\beta$, IL-4, IL-6, IL-8, TNF- $\alpha$, TGF- $\alpha$, and HB-EGF as potential candidates for mediating pyocyanin-triggered MUC5AC release.

\section{IL-1 $\beta$, TGF- $\alpha$, and TNF- $\alpha$ mediate pyocyanin-induced MUC5AC release}

We found that inflammatory mediators such as TGF- $\alpha$, TNF- $\alpha$ and IL- $1 \beta$ were strong inducers of MUC5AC release, whereas the response to IL-6 was weak (Figure 4e). ATP, which has been shown to trigger mucin production in TBECs, ${ }^{19}$ produced weak MUC5AC release (Figure 4e). Although induced in TBECs exposed to pyocyanin, IL- $1 \alpha$, IL-4, and IL- 8 do not initiate mucin secretion and thus probably do not mediate the toxin's effects (Figure 4e). In contrast, EGF is an excellent stimulus of mucin production in these cells (Figure 4e), although it is not released upon exposure to pyocyanin (Figure $4 \mathbf{a}$ and $\mathbf{d}$ ) and does not appear to participate in pyocyanin-mediated mucin production. MUC5AC induction by IL- $1 \beta$, TGF- $\alpha$, TNF- $\alpha$, or IL-6 was completely blocked by the EGFR inhibitor (AG1478), but not by the control inhibitor analog (AG9) (Figure 4e). Thus, IL-1 $\beta$, IL-6, TGF- $\alpha$, and TNF- $\alpha$ appear to signal upstream of EGFR to induce release of MUC5AC.

We used neutralizing antibodies against IL- $1 \beta$, IL- 6 , TGF- $\alpha$, and TNF- $\alpha$ to confirm their involvement in the process. All of these antibodies exhibited inhibitory effects on pyocyanininduced MUC5AC release, although inhibition by anti-IL- 6 did not reach significant levels (Figure 4f). Elimination of extracellular ATP by apyrase had no effect (Figure 4f), consistent with its low potency in inducing mucin release (Figure 4e). As TNF$\alpha$ was shown to increase EGFR expression, we tested whether TNF- $\alpha$ potentiates pyocyanin-induced MUC5AC release. Concentrations of TNF- $\alpha$ found in supernatants of pyocyanintreated epithelial cells $(<0.5 \mathrm{ng} / \mathrm{ml}$, Figure $4 \mathbf{d})$ increased both EGFR expression and pyocyanin-induced MUC5AC release (Figure 4g). These results support a mechanism in which pyocyanin acts through multiple autocrine/paracrine signals (IL-1 $\beta$, TGF- $\alpha$, TNF- $\alpha$ ) that activate EGFR, leading to mucin secretion in TBECs.

\section{Gene expression changes in response to pyocyanin}

Comparison of genome-wide expression profiles in pyocyanin-exposed and unexposed cells revealed robust changes (Supplementary Figure S3 online). Conservative thresholds of fourfold change and $P$ value $<10^{-3}$ yielded 268 genes (unique Entrez IDs) modified by pyocyanin (Supplementary Figure S3 online), including some previously reported genes mediating mucin synthesis (pyocyanin-upregulated genes, Supplementary Table S1 online; pyocyanin-downregulated genes, Supplementary Table S2 online). Gene annotationbased clustering of pyocyanin-responsive genes revealed 11 clusters containing at least 5 genes (Figure 5). One major group of biological functions (clusters $6,7,8,11$ ) were related to cell migration and responses to stimuli (inflammatory/ wound response, chemotaxis, cell migration, wound healing) (Table 1 and Supplementary Table $\mathbf{S} 3$ online). Other groups (clusters 1 and 2) contain genes involved in cell proliferation and positive regulation of gene expression. A third group of functions were associated with apoptosis (clusters 3, 9, 10). Cluster 8 subsumes many of the functions involved in stress responses, including responses to oxidative stress and ROS (Supplementary Table S3 online). Ingenuity pathway analysis (IPA v.7.6) identified and ranked signaling networks with the highest statistical associations among pyocyanin-responsive genes. Network number 1 was identified as genes associated with TNF- $\alpha$ (Supplementary Figure S4 online), whereas the second highest involves genes associated with nuclear factor (NF) $-\kappa B$ (Supplementary Figure S5 online). These data uncover strong transcriptional relationships in airway 


\section{ARTICLES}

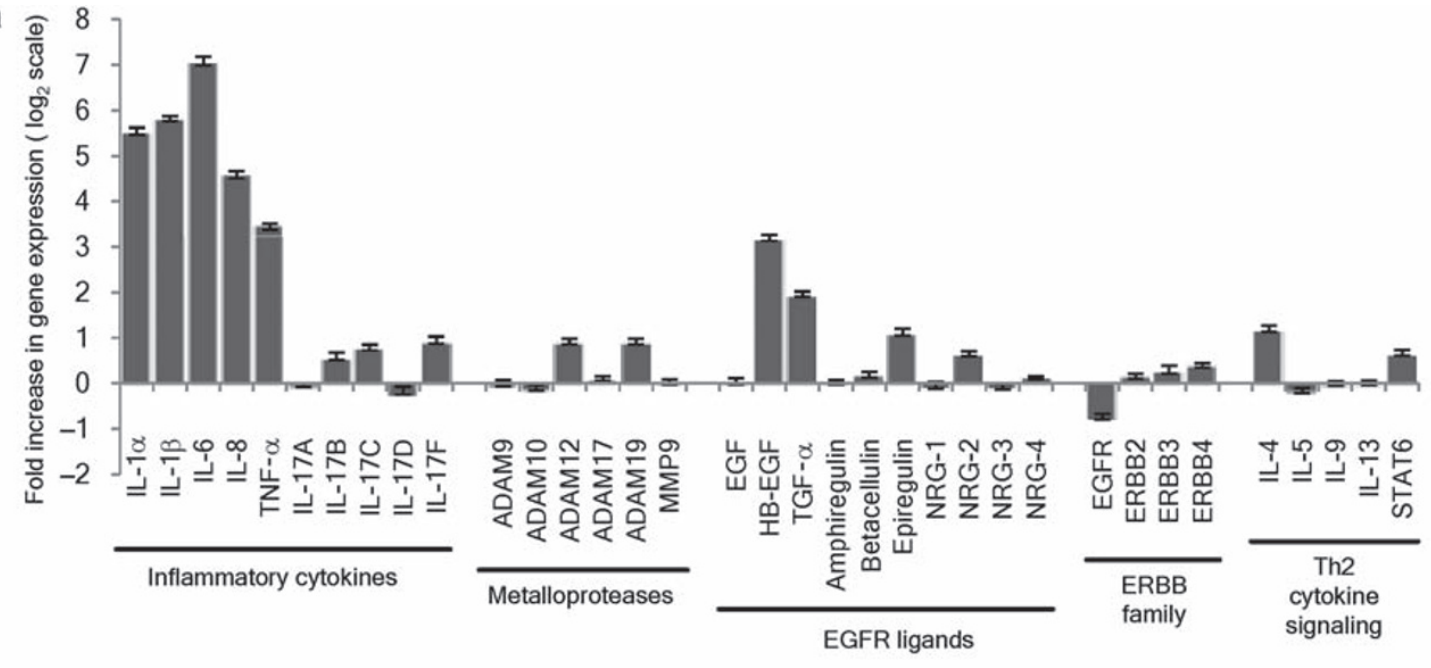

b

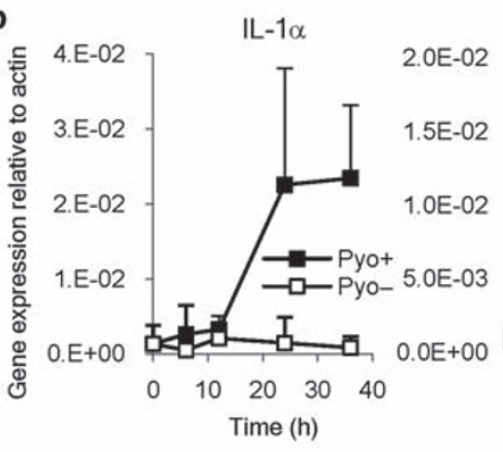

c

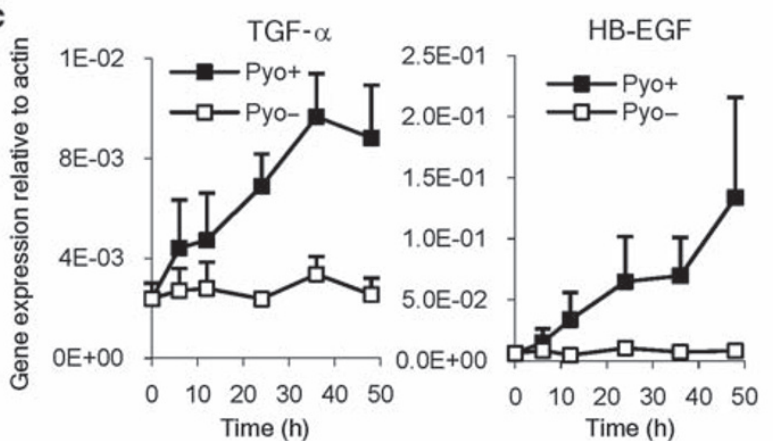

e

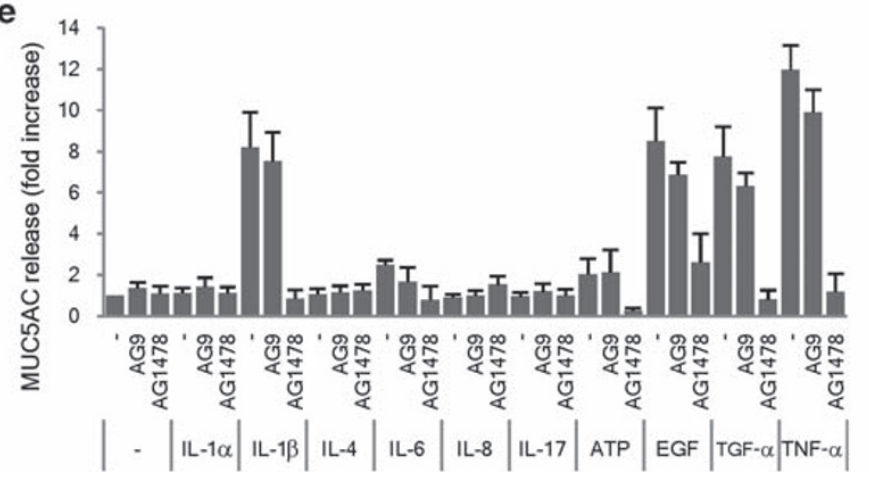

IL-1 $\beta$

IL-6

TNF- $\alpha$
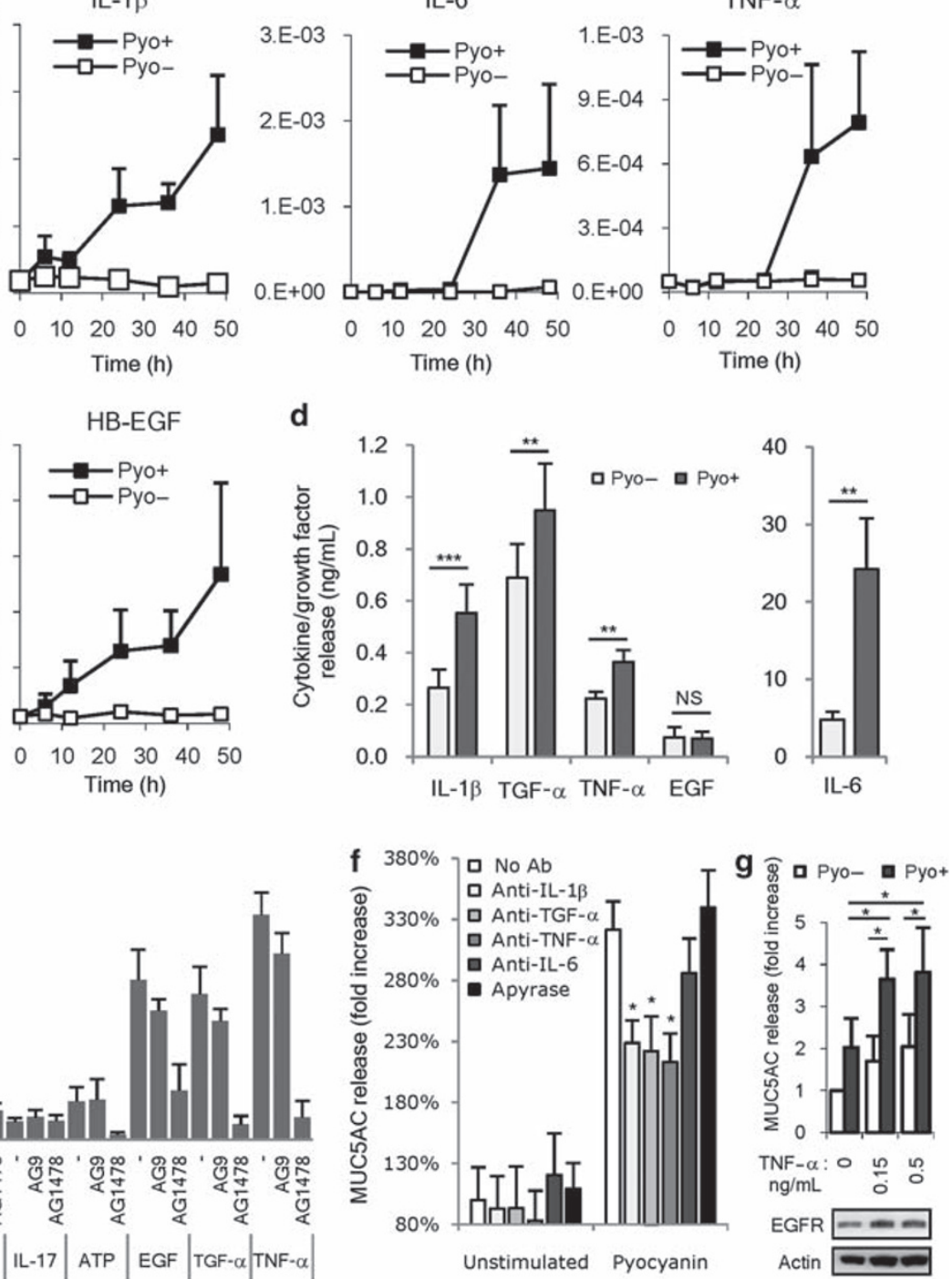
epithelial cells in response to pyocyanin, many of which can be primary or secondary consequences of exposure to ROS.

\section{Pyocyanin induces epithelial transcription of inflammatory mediators reported at elevated levels in CF patients}

To gain insight into the relevance of pyocyanin's effects on TBECs in contributing to the inflammatory conditions of Pseudomonas-infected airways, we asked whether pyocyanin-induced transcriptional changes detected by microarray are consistent with inflammatory mediators known to be elevated in CF patients. Correlations with genes upregulated in CF would suggest a novel mechanism in which pyocyanin-induced epithelial release would account for their gross increased levels in CF patients.

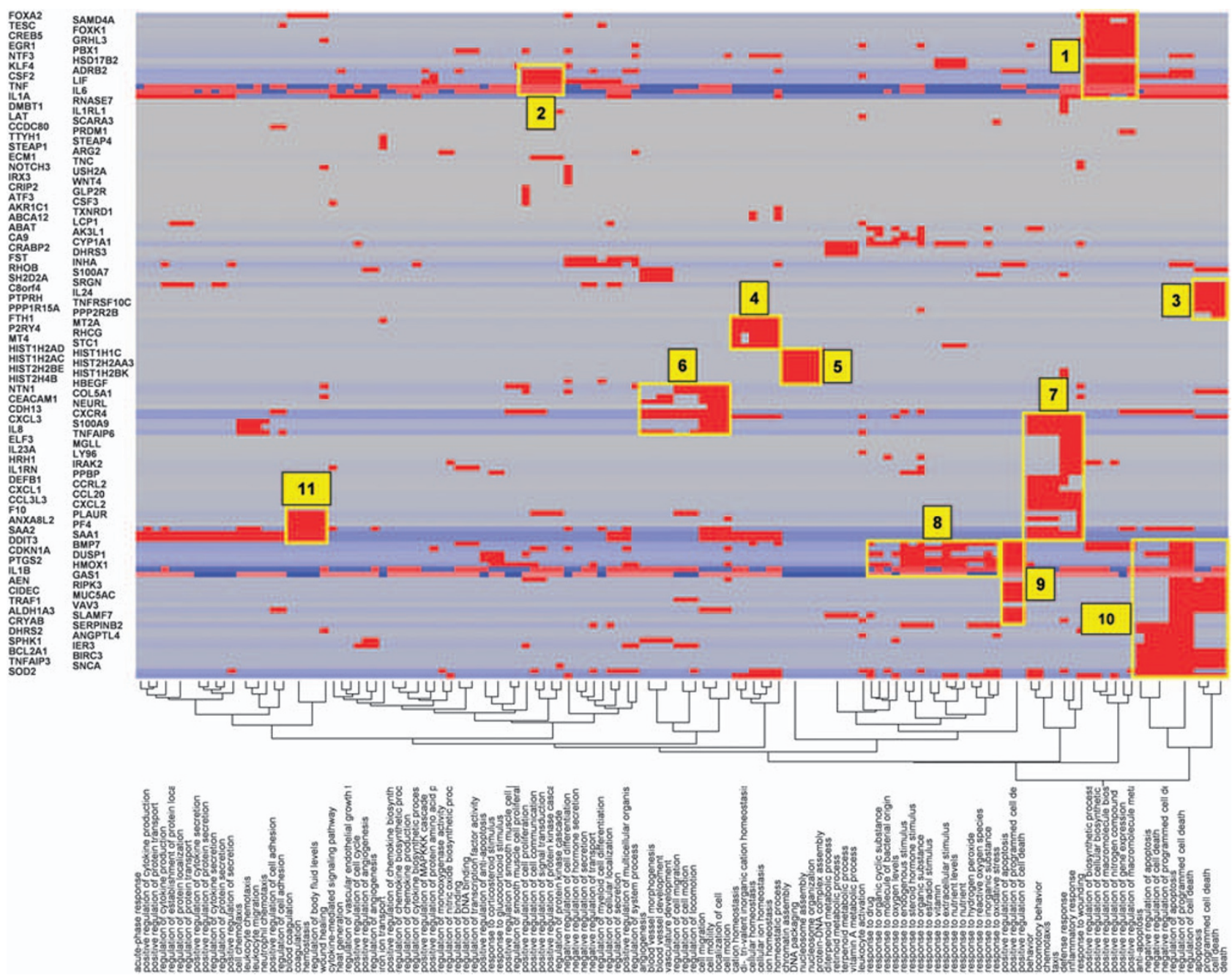

Figure 5 Functional clustering of pyocyanin-responsive genes in airway epithelial cells. The heat map shows a two-way clustering of genes (vertical axis) and the associated GO FAT functions (horizontal axis). Red cells indicate assignment of an altered gene to a function. (Due to data standardization, genes that participate in many functions, such as tumor necrosis factor (TNF), interleukin (IL)-1 $\alpha$, IL-1 $\beta$, and IL6, produce pinkish cells.) Clusters encompassing at least five genes were selected visually and numbered arbitrarily from 1-11. The clustered genes and corresponding functions are listed in Table 1 and Supplementary Table S3 online. The data are obtained from H292 cells exposed to $8 \mu \mathrm{M}$ pyocyanin for $48 \mathrm{~h}$.

Figure 4 Pyocyanin-induced transcription of inflammatory cytokines and epidermal growth factor receptor (EGFR) ligands mediate MUC5AC secretion. (a) Pyocyanin-induced transcriptional changes for genes known as inducers of mucin synthesis or involved in their signaling in bronchiolar epithelial cells assayed by microarray. Transcription of interleukin (IL)-1 $\alpha$, IL-1 $\beta$, IL-6, IL-8, tumor necrosis factor-alpha (TNF- $\alpha$ ), heparin-bound EGF (HB-EGF), and tissue growth factor-alpha (TGF- $\alpha$ ) were strongly induced by pyocyanin. (b) Pyocyanin-induced transcriptional changes of the inflammatory cytokines IL-1 $\alpha$, IL-1 $\beta$, IL-6 and TNF- $\alpha$ were followed over time by real-time reverse transcriptase (RT)-PCR (mean \pm s.e.m., $n=3-5)$. (c) Transcriptional changes of the EGFR ligands, TGF- $\alpha$, and HB-EGF were confirmed by real-time RT-PCR (mean \pm s.e.m., $n=3-5$ ). (d) IL-1 $\beta$, TGF- $\alpha$, TNF- $\alpha$, EGF, and IL- 6 protein levels were determined in supernatants of pyocyanin-treated cells (mean \pm s.e.m., $n=4)$. (e) MUC5AC release from cells exposed to a variety of stimuli was measured in the presence of the EGFR inhibitor (AG1478) or its control compound (AG9) (mean \pm s.e.m., $n=4$ ). Concentration of cytokines/growth factors: $10 \mathrm{ng} / \mathrm{ml},[$ ATP] $=100 \mu \mathrm{M}$. (f) Neutralizing antibodies against IL-1 $\beta$, IL-6, TNF- $\alpha$, and TGF- $\alpha$ inhibit MUC5AC release from pyocyanin-treated bronchiolar epithelial cells (mean \pm s.e.m., $n=7-12$ ). (g) Pretreatment of $\mathrm{H} 292$ cells with TNF- $\alpha$ (2 days) increases EGFR levels and further potentiates pyocyanin-stimulated MUC5AC release (mean \pm s.e.m., $n=4$. (pyocyanin) $=4 \mu \mathrm{M}, 2$ days ${ }^{\star} P<0.05 ;{ }^{* \star} P<0.01 ;{ }^{* \star *} P<0.001$ ). Pyo, pyocyanin. 
Table 1 Summary of gene-function clusters

\begin{tabular}{ll}
\hline Cluster & Functions \\
\hline C3 (3.34) & Apoptosis \\
C7 (3.33) & $\begin{array}{l}\text { Inflammatory/wound response, } \\
\text { chemotaxis }\end{array}$ \\
& \\
C11 (3.29) & Hemostasis wound healing \\
C2 (2.94) & Positive regulation of cell proliferation \\
$\mathbf{C 8}(2.86)$ & Response to stimuli \\
C6 (2.76) & Angiogenesis, cell migration \\
C10(2.73) & Antiapoptosis, apoptosis
\end{tabular}

\section{Genes (log ratio)}

C8orf4 (3.50), IL24 (6.87), PPP1R15A (3.34), PPP2R2B (-2.78), PTPRH (4.25), SRGN (2.53), TNFRSF10C (2.01)

ANXA8L2 (-2.14), CCL20 (5.88), CCL3L3 (2.08), CCRL2 (2.00), CXCL1 (5.07), CXCL2 (4.56), CXCL3 (5.04), CXCR4 (4.37), DEFB1 (-3.33), ELF3 (3.44), F10 (3.66), HRH1 (2.03), IL1RN (2.49), IL23A (3.85), IL8 (4.51), IRAK2 (2.43), LY96 (2.55), MGLL (2.17), PF4 (3.34), PLAUR (2.46), PPBP (3.48), S100A9 (2.34), SAA1 (3.24), SAA2 (3.45), TNFAIP6 (2.58)

ANXA8L2 (-2.14), F10 (3.66), PF4 (3.34), PLAUR (2.46), SAA1 (3.24), SAA2 (3.45)

ADRB2 (2.21), CSF2 (2.44), IL6 (6.82), LIF (2.94), TNF (3.38)

BMP7 (-2.00), CDKN1A (3.41), DDIT3 (2.24), DUSP1 (2.73), HMOX1 (2.86), IL 1B (5.71), PTGS2 (3.93)

CDH13 (2.55), CEACAM1 (2.87), COL5A1 (-2.65), CXCL3 (5.04), CXCR4 (4.37), HBEGF (3.00), IL8 (4.51), NEURL (2.34), NTN1 (2.30), S100A9 (2.34)

AEN (2.03), ALDH1A3 (2.34), ANGPTL4 (2.69), BCL2A1 (4.90), BIRC3 (3.41), BMP7 (-2.00), CDKN1A (3.41), CIDEC (2.11), CRYAB (-3.63), DDIT3 (2.24), DHRS2 (2.16), DUSP1 (2.73), GAS1 (-4.10), HMOX1 (2.86), IER3 (2.86), IL1B (5.71), MUC5AC (4.11), PTGS2 (3.93), RIPK3 (2.73), SERPINB2 (3.72), SLAMF7 (2.19), SNCA (-2.06), SOD2 (2.88), SPHK1 (2.02), TNFAIP3 (2.99), TRAF1 (2.53), $\operatorname{VAV} 3(-2.73)$

AEN (2.03), ALDH1A3 (2.34), BMP7 (-2.00), CDKN1A (3.41), CIDEC (2.11), DDIT3 (2.24), DUSP1 (2.73), HMOX1 (2.86), IL1B (5.71), MUC5AC (4.11), PTGS2 (3.93), RIPK3 (2.73), SLAMF7 (2.19), VAV3 (-2.73)

HIST1H1C (2.92), HIST1H2AC (2.75), HIST1H2AD (2.12), HIST1H2BK (3.16), HIST2H2AA3 (2.61), HIST2H2BE (2.52), HIST2H4B (2.18)

ADRB2 (2.21), CREB5 (5.20), CSF2 (2.44), EGR1 (-2.04), FOXA2 (2.59), FOXK1 (2.09), GRHL3 (2.22), IL 1A (5.32), IL6 (6.82), KLF4 (2.13), LIF (2.94), NTF3 (2.27), PBX1 (-2.54), SAMD4A (2.89), TESC (2.10), TNF (3.38)

FTH1 (2.13), MT2A (2.05), MT4 (2.73), P2RY4 (3.27), RHCG (2.27), STC1 (2.17)

\section{C4 (2.22)}

Cation homeostasis

Clusters are numbered as in Figure $\mathbf{5}$ and ordered by the median absolute value of the log ratios for the cluster members (in parentheses). Log ${ }_{2}$ ratios for individual genes are shown for pyocyanin treated vs. untreated. Downregulated genes are shown in bold. The individual GO FAT functions constituting each cluster are given in Supplementary Table $\mathbf{S} \mathbf{3}$ online. Data are obtained from cells exposed to $8 \mu \mathrm{M}$ pyocyanin.
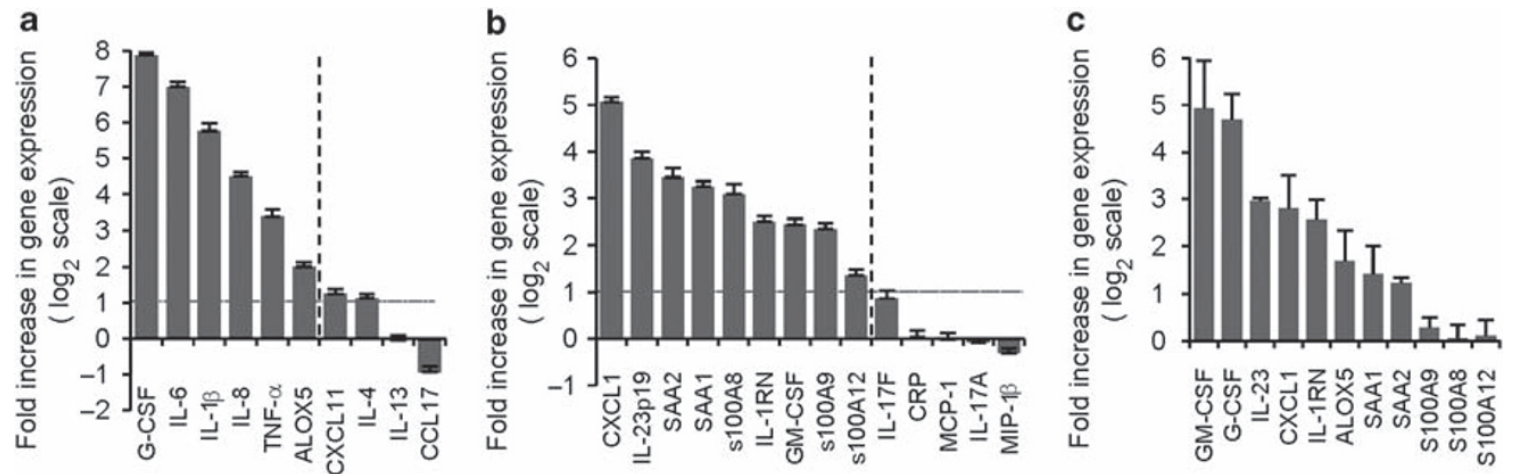

Figure 6 Pyocyanin induces epithelial transcription of several inflammatory mediators reported as elevated in cystic fibrosis (CF) patients. Gene expression changes in pyocyanin-treated epithelial cells $(48 \mathrm{~h}, 8 \mu \mathrm{M})$ are shown for 24 inflammatory mediators (cytokines, chemokines, or acute-phase proteins) previously reported as displaying increased levels in CF patients (Supplementary Table S4 online). (a) Six of ten genes specifically associated with Pseudomonas infection in CF are induced at least twofold by pyocyanin in bronchiolar epithelial cells (microarray data). (b) Nine of fourteen additional CF-associated genes (not studied in relation to Pseudomonas) were also induced by pyocyanin in bronchiolar epithelial cells (microarray data). (c) Real-time PCR confirmation of gene expression changes of CF-associated genes upregulated by pyocyanin. Quantitative PCR results were not only obtained from samples used for microarray but from additional, independent experiments as well ( $n=4-9)$. CXCL1, chemokine (C-X-C motif) ligand 1; CXCL11, chemokine (C-X-C motif) ligand 11; G-CSF, granulocyte colony-stimulating factor; GM-CSF, granulocyte-monocyte colony-stimulating factor; IL, interleukin; TNF- $\alpha$, tumor necrosis factor-alpha.

From a survey of medical literature (reports strictly on human subjects, no animal model, or cell culture studies) we assembled a list of 24 inflammatory mediators elevated in CF patients (sputum, serum, or bronchoalveolar lavage fluid) (Supplementary Table S4 online). We subdivided these genes into two groups based on the focus of the study: (1) Four papers compared cytokine and chemokine levels between CF patients with or without $P$. aeruginosa infection 
(Supplementary Table S4 online, list A). These reports showed 10 inflammatory mediators at significantly elevated levels in CF patients due to Pseudomonas infection: chemokine (C-C motif) ligand 17 (CCL17), chemokine (C-X-C motif) ligand 11 (CXCL11), IL-1 $\beta$, IL-4, IL-6, IL-8, IL-13, granulocyte colony-stimulating factor (G-CSF, CSF3), leukotriene B4 (LTB4), and TNF- $\alpha$. (2) The second group reported additional CF-associated inflammatory mediators whose correlation with Pseudomonas infection was not specified (Supplementary Table S4 online, list B). This list comprised 14 genes detected at increased levels in CF patients: chemokine (C-X-C motif) ligand 1 (CXCL1), IL-23, serum amyloid (SAA) 1 and 2, S100A8, S100A9, S100A12, IL-1 receptor antagonist (IL-1RN), granulocyte-monocyte colony stimulating factor (GM-CSF, CSF2), IL-17F, C-reactive protein, MCP-1, and MIP-1 $\beta$. Our microarray data confirmed by real-time PCR shows that 6 of 10 pseudomonas associated inflammatory mediators (G-CSF, IL-6, IL- $1 \beta$, IL-8, TNF- $\alpha$, LTB4) and 6 of 14 additional CF-associated genes (GM-CSF, CXCL1, IL-23, IL-1Ra, SAA1, SAA2) were upregulated in TBECs by pyocyanin (Figure 6a-c). This suggests that pyocyanin's effect on bronchiolar epithelial cells can be a major contributor to the initiation and maintainance of the hyperinflammatory state in CF airways.

\section{Pyocyanin induces epithelial transcription of other genes} involved in innate immunity and response to oxidative stress Among the pyocyanin-upregulated genes, we identified several ILs and chemokines known to have important roles in the innate or adaptive immune system (Table 2). IL-11, -19, and -20 showed modest changes in transcription, whereas IL-24 showed robust upregulation (Table 2). Beyond ILs, pyocyanin strongly induced transcription of one IL receptor (IL 1 receptor type 2 (IL1R2)), three chemokines (CXCL2, CXCL3, CCL20), and one chemokine receptor (CXCR4) (Table 2).

Pyocyanin imposes oxidative stress on cells by generating intracellular ROS. We identified 10 genes related to oxidative stress induced by pyocyanin (Table 2). Real-time PCR confirmed induction of mitochondrial SOD (SOD2) by pyocyanin (8.5-fold), whereas transcript levels of SOD1 (1.01 \pm 0.23 -fold, $n=3)$ and SOD3 $(0.82 \pm 0.20, n=3)$ remained unchanged. Catalase activity was reported to be inhibited by pyocyanin. ${ }^{30}$ We found slightly decreased catalase transcript levels upon pyocyanin exposure $(0.63 \pm 0.21$-fold, $n=3)$. Growth differentiation

Table 2 Transcriptional changes of novel pyocyanin-responsive genes involved in airway inflammation and responses to oxidative stress in airway epithelial cells

\begin{tabular}{|c|c|c|c|}
\hline Gene symbol & Gene name & Microarray & qPCR \\
\hline$I L-11$ & Interleukin-11 & 2.7 & 23.0 \\
\hline IL-20 & Interlekuin-20 & 3.6 & 21.0 \\
\hline IL-24 & Interleukin-24, transcript variant 2 & 128.6 & 1601.0 \\
\hline IL-1R2 & Chemokines, chemokine receptors & & \\
\hline CXCL2 & Chemokine ( $\mathrm{C}-\mathrm{X}-\mathrm{C}$ motif) ligand 2 & 34.5 & 40.1 \\
\hline CXCL3 & Chemokine ( $\mathrm{C}-\mathrm{X}-\mathrm{C}$ motif) ligand 3 & 50.8 & 38.9 \\
\hline CCL20 & Chemokine (C-C motif) ligand 20 & 58.7 & 53.4 \\
\hline CXCR4 & Chemokine ( $\mathrm{C}-\mathrm{X}-\mathrm{C}$ motif) receptor 4 & 22.0 & 115.2 \\
\hline$S N C A$ & Synuclein, alpha (non-A4 component of amyloid precursor) & 0.267 & 0.076 \\
\hline DHRS2 & Short-chain dehydrogenase/reductase 2 & 4.5 & 3.0 \\
\hline DDIT3 & DNA-damage-inducible transcript 3 & 4.7 & 9.1 \\
\hline DUSP1 & Dual specificity phosphatase 1 & 6.6 & 5.0 \\
\hline HMOX1 & Heme oxygenase (decycling) 1 & 7.3 & 26.1 \\
\hline SOD2 & Superoxide dismutase 2 , mitochondrial & 7.4 & 8.5 \\
\hline S100A7 & S100 calcium binding protein A7 & 10.8 & 4.2 \\
\hline PTGS2 & Prostaglandin-endoperoxide synthase 2 & 16.0 & 4.4 \\
\hline
\end{tabular}

Abbreviation: qPCR, quantitative PCR.

Microarray: fold changes, mean, $n=4$. qPCR: fold changes, mean, $n=3-5$.

QPCR results were not only obtained from samples used for microarray but from additional, independent experiments as well.

Bronchiolar epithelial cells were exposed to $8 \mu \mathrm{M}$ pyocyanin for $48 \mathrm{~h}$. 
factor-15 (GDF-15), a stress-induced cytokine, was strongly upregulated by pyocyanin (fold increases: microarray, 70.0-fold; real-time PCR, 256.1-fold $(n=3))$.

\section{DISCUSSION}

Obstruction of the airways due to mucus overproduction is characteristic of several chronic respiratory diseases (CF, asthma, chronic obstructive pulmonary disease).${ }^{18}$ In addition to increased mucus production in CF respiratory epithelial cells, P. aeruginosa also elicits mucus secretion. ${ }^{18}$ Pseudomonas culture supernatants, lipopolysaccharide, and homoserine-lactone have been shown to induce MUC5AC or MUC2 in airway epithelial cells. ${ }^{28,31,32}$ We show for the first time that TBECs respond to pyocyanin with acute mucin secretion (MUC5AC release and MUC2 induction). We hypothesize that this mechanism contributes to mucus overproduction seen in Pseudomonas-infected airways (Figure 7). We found that intracellular production of ROS is responsible for pyocyanin-induced mucin secretion. Although oxidative stress of different origins (bolus $\mathrm{H}_{2} \mathrm{O}_{2}$, Nox4, Duox1) was already associated with MUC5AC production, our observations are the first describing ROS-initiated transcription of MUC2. ${ }^{21,22,33}$

Several different surface stimuli converge by signaling through EGFR and activate common innate immune mechanisms. ${ }^{24,26,34}$ We show evidence that MUC2 and MUC5AC induction by pyocyanin occurs entirely through activation of the EGFR signaling pathway (EGFR-MEK1/2-ERK) and that pyocyanin also promotes epithelial cell proliferation via EGFR (Figure 7). Pyocyanin was shown recently to induce Goblet cell hyperplasia in mouse lungs in vivo. ${ }^{11} \mathrm{~A}$ two-step process was proposed as the mechanism for in vivo trans-differentiation of Goblet cells from ciliated epithelial cells. ${ }^{23}$ First, activation of EGFR signaling was suggested to promote cell proliferation in ciliated airway epithelial cells, allowing the second, subsequent initiation of Goblet cell differentiation. ${ }^{23}$ Our data support this model by showing a prominent role of ligand-activated (autocrine) EGFR signaling in response to pyocyanin.

Inhibiting de novo protein synthesis blocked pyocyanininduced transcription of MUC2 and MUC5AC, suggesting that EGFR is activated predominantly in a ligand-dependent manner, although we cannot rule out concurrent ligand-independent activation, such as ROS-mediated inhibition of intracellular phosphatases (Figure 7). ${ }^{26}$ Such ligand-independent EGFR activation could involve excess intracellular ROS produced by pyocyanin, as we observed inhibitory effects of intracellular antioxidants on mucin induction and secretion (Figure $2 \mathbf{c}, \mathbf{d}$ ). We identified several secreted cytokines and EGFR ligands that mediate pyocyanin-induced MUC5AC release: IL-1 $\beta$, IL-6, TGF- $\alpha$, TNF- $\alpha$, and HB-EGF. These inflammatory mediators are known to trigger MUC5AC production in TBECs, and we found they were released upon exposure to pyocyanin. ${ }^{18} \mathrm{To}$ our knowledge, this is the first demonstration that MUC5AC release induced by IL- $1 \beta$ or IL- 6 is EGFR dependent. The exact mechanism is unknown but transactivation of EGFR by IL-1 $\beta$ or IL-6 is a possibility, as EGFR transactivation by various stimuli was already reported. ${ }^{24,33,35}$ Our work describes for the first

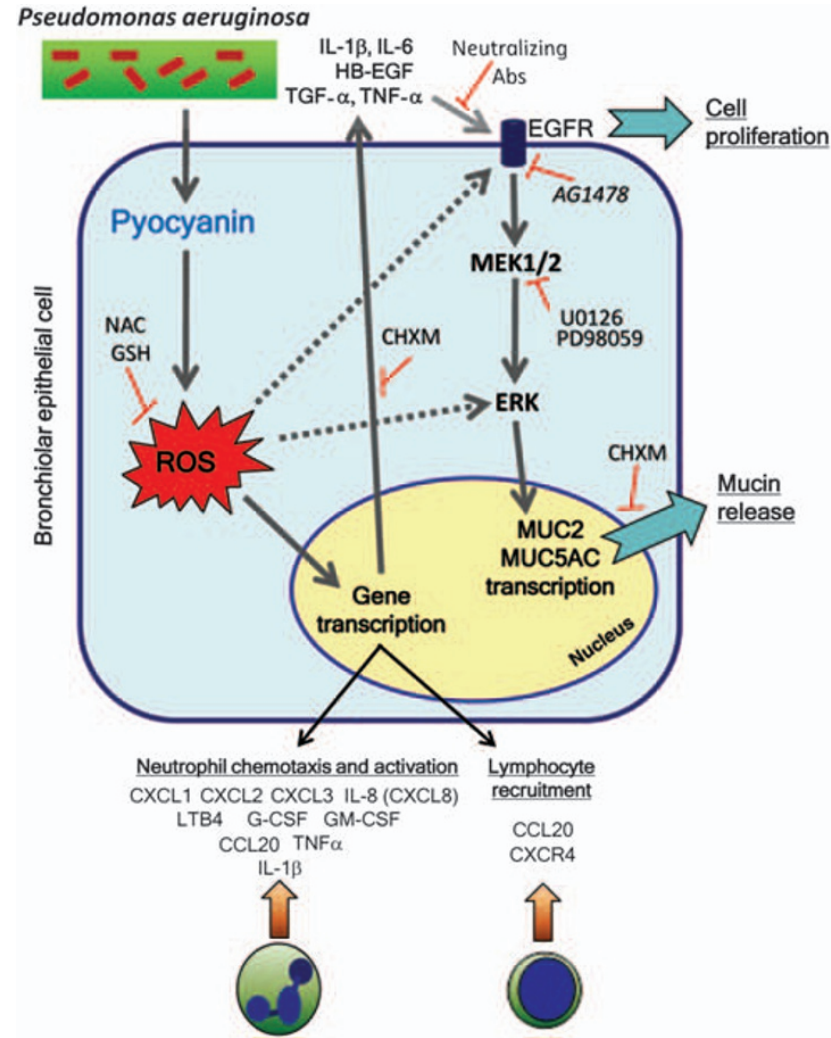

Figure 7 Proposed model of pyocyanin's inflammatory action in airway epithelial cells. The scheme shows proposed mechanisms for pyocyanin-induced mucin release and transcriptional changes. Reactive oxygen species (ROS) produced in the host cell by pyocyanin result in transcriptional changes of several genes involved in the host's response to oxidative stress and in the recruitment of white blood cells to the infected mucosa. Inductions of tumor necrosis factor-alpha (TNF- $\alpha$ ), interleukin (IL)-1 $\beta$, tissue growth factor-alpha (TGF- $\alpha$ ), IL-6, and heparin-bound epidermal growth factor (HB-EGF) collectively lead to activation of EGF receptor (EGFR), followed by activation of MEK1/2, extracellularsignal-regulated kinase (ERK), MUC2/MUC5AC transcription, and cell proliferation. Red lines indicate points of experimental intervention with inhibitors, ROS scavengers, and neutralizing antibodies. Abs, antibodies; CCL20, chemokine (C-C motif) ligand 20; CHXM, cycloheximide; CXCL1, 2, 3, chemokine (C-X-C motif) ligands 1, 2, and 3; NAC, N-acetyl cysteine.

time increased transcription of two EGFR ligands, TGF- $\alpha$ and HB-EGF, by pyocyanin. TGF- $\alpha$ shedding by ADAM17 was previously shown to be stimulated by ROS. ${ }^{22}$ Pyocyanin-induced increases in MUC2 transcription could be mediated by TNF- $\alpha$, IL-1 $\beta$, TGF- $\alpha$, and HB-EGF, as these stimuli are known inducers of MUC2 transcription and we showed they are released from airway cells by pyocyanin. ${ }^{35-38}$ Thus, we propose that a complex network of autocrine signals mediate pyocyanin-induced mucin production in TBECs (Figure 7).

Pyocyanin has been suggested to have proinflammatory effects on airway epithelial cells but the underlying mechanisms are poorly understood. ${ }^{6}$ Previously, airway epithelial cells were shown to produce IL- 8 in response to pyocyanin..$^{29}$ Beyond confirming increased IL-8 expression, we detected enhanced production of additional inflammatory cytokines: IL-1 $\beta$, IL-6, and TNF- $\alpha$, all of which were detected at elevated levels in 
CF patients and were associated with Pseudomonas infection (Figure 6a). They are induced by oxidative stress, their downstream signaling involves ROS, and they are regulated by the transcription factor NF- $\kappa \mathrm{B} .{ }^{39,40}$ Pyocyanin-responsive genes were significantly associated with NF- $\mathrm{\kappa B}$ (network number 2, Supplementary Figure S5 online), suggesting that ROSdependent activation of NF- $\kappa \mathrm{B}$ is important in the release of these cytokines.

TNF- $\alpha$ is a multifunctional proinflammatory cytokine. Increased concentrations of TNF- $\alpha$ have been reported in CF bronchoalveolar lavage fluid, and TNF- $\alpha$ levels in CF sputum are negatively correlated with pulmonary function (Supplementary Table S4 online). Ingenuity pathway analysis of our microarray findings identified TNF- $\alpha$-associated genes as the statistically most significant network among pyocyanin-responsive genes (Supplementary Figure S4 online). Pyocyanin-induced epithelial TNF- $\alpha$ release can induce several secondary genes (including MUC5AC, MUC2, GM-CSF). TNF- $\alpha$ is a potent neutrophilpriming and -activating agent. ${ }^{24}$ Pyocyanin-induced epithelial release of IL- $1 \beta$ is important in mediating mucus overproduction and can contribute to initiation of inflammatory responses. Upon exposure to pyocyanin, airway epithelial cells experienced more than a fivefold increase in IL- 6 secretion. IL- 6 has both pro- and anti-inflammatory properties. ${ }^{39}$ Increased IL- 6 release by pyocyanin could contribute to the acute-phase reaction and mucus overproduction but at the same time it could limit the extent of the inflammatory response by inducing the transcription of the natural IL-1 inhibitor, IL-1R antagonist (IL-1Ra), and by reducing IL- $1 \beta$ production. ${ }^{39}$ Indeed, increase in IL- 6 transcript levels is delayed compared with that of IL- $1 \beta$ mRNA levels (Figure $\mathbf{4 b}$ ) and IL-1R antagonist transcript (IL1RN) levels are also enhanced (Figure $\mathbf{6 b}, \mathbf{c}$ ).

Pseudomonas-infected CF patients have higher serum G-CSF levels than CF patients without Pseudomonas infection (Supplementary Table S4 online). Airway epithelial cells produce G-CSF and GM-CSF when exposed to P. aeruginosa. ${ }^{41}$ We show for the first time that pyocyanin induces robust increases in G-CSF and in GM-CSF transcription in TBECs. G-CSF could contribute significantly to CF disease severity as it is a potent neutrophil chemoattractant (Figure 7), promotes neutrophil survival by inhibiting apoptosis, and increases neutrophil release from the bone marrow. ${ }^{42}$ GM-CSF is important in the pulmonary immune system, as it also promotes neutrophil maturation, activation, and survival. ${ }^{41,43}$

LTB4 is a potent neutrophil chemoattractant seen at elevated levels in CF patients' sputum, saliva, and bronchoalveolar lavage fluid (Supplementary Table S4 online). LTB4 is generated from arachidonic acid by the enzyme lipooxygenase. TBECs are capable of synthesizing LTB $4 .{ }^{44}$ Our microarray findings detected increased expression of ALOX5, the gene coding lipooxygenase, in pyocyanin-exposed TBECs (Figure 6a). This suggests that increased epithelial LTB4 production by pyocyanin could contribute to neutrophil recruitment in Pseudomonas-infected airways.

We found several chemokines (CXCL1, CXCL2, CXCL3, CXCL8, CCL20) that were induced by pyocyanin in epithelial cells (Figure 6b, Table 2). CXCL1 expression was increased the most. Increased CXCL1 levels have been associated with CF (Supplementary Table S4 online). The CXC-chemokines (CXCL1-3 and CXCL8 (IL-8)) attract neutrophils; their increased epithelial expression could contribute to neutrophil infiltration in CF airways. CCL20 is strongly chemotactic for lymphocytes, weakly for neutrophils. ${ }^{45}$ CXCR4 and CCL20 expression were reported in human airway epithelial cells; their increase by pyocyanin could have a role in enhanced lymphocyte recruitment (Figure 7). ${ }^{45}$

Pyocyanin also boosted transcription of the acute-phase proteins, SAA1 and 2 (Figure 6b). Increased levels of SAAs were detected in CF patients' serum (Supplementary Table S4 online). SAA release was detected in airway epithelial cells. ${ }^{46}$ Pyocyanin-induced epithelial SAA transcription could be mediated by IL- $1 \beta$, IL- 6 , or TNF- $\alpha$ as these proinflammatory mediators are known to induce SAA secretion in the liver. ${ }^{46}$

Table 2 presents four ILs (IL-11, -19, -20, and -24) found to be upregulated by pyocyanin in TBECs that have not been previously associated with $\mathrm{CF}$ and their function in Pseudomonas respiratory infections has not been elucidated. The robust induction of IL-24 is especially remarkable. Pyocyanin-induced expression of IL-23 in TBECs might be important as IL-23 has been associated with CF (Figure $\mathbf{6 b}$ ) and was shown to mediate inflammatory responses in mouse lung infected with $P$. aeruginosa. ${ }^{47}$ The lower part of Table 2 lists pyocyanin-modified genes associated with oxidative stress, consistent with its wellknown pro-oxidant effects. Three genes (CRYAB, SCARA3, SNCA) were downregulated, whereas seven (DHRS2, DDIT3, DUSP1, HMOX1, SOD2, S100A7, and PTGS2) were upregulated (Table 2).

$P$. aeruginosa requires pyocyanin for full virulence in a variety of infection models. The majority of Pseudomonas strains infecting human airways secrete this toxin. Despite its in vivo significance, little is known about how this toxin affects airway epithelial cells. Our work uncovered numerous novel aspects of pyocyanin's proinflammatory action on the respiratory epithelium, as many of the characteristic features of CF lung disease (mucus overproduction, proinflammatory cytokine release and induction of inflammatory mediators involved in leukocyte recruitment) could be reproduced by exposing bronchiolar epithelial cells to pyocyanin. Our data consolidates the crucial role of airway epithelial cells in initiation and maintenance of the airway's immune and inflammatory response to $P$. aeruginosa and emphasizes the importance of the complex consequences of oxidative stress caused by this remarkable bacterial toxin.

\section{METHODS}

Airway epithelial cells. The human mucoid bronchial epithelial cell line, H292, was purchased from American Type Culture Collection (Manassas, VA). Cells were grown in RPMI-1640 medium (Invitrogen, Carlsbad, CA) containing 10\% fetal bovine serum (FBS) (Hyclone, Logan, UT), $1 \%$ penicillin-streptomycin, $1 \%$ L-glutamine, $1 \%$ sodium pyruvate, and 1\% HEPES (Invitrogen). The immortalized human bronchiolar epithelial cells, 16HBE140-, were obtained from Dr Gruenert. ${ }^{48}$ Cells were cultured on six-well tissue culture plates coated with fibronectin-coating solution ( $1 \%$ bovine serum albumin, $0.03 \mathrm{mg} / \mathrm{ml}$ bovine 
collagen, and $0.01 \mathrm{mg} / \mathrm{ml}$ human fibronectin) in minimum essential medium (MEM) medium containing L-glutamine, glucose, $\mathrm{NaHCO}$, $10 \%$ FBS, and $1 \%$ penicillin-streptomycin (Invitrogen). IB3-1 cells and BEAS-2B cells were purchased from American Type Culture Collection. IB3- 1 cells were maintained in MEM medium containing L-glutamine, glucose, $10 \% \mathrm{FBS}$, and $1 \%$ penicillin-streptomycin (Invitrogen). BEAS$2 \mathrm{~B}$ cells were cultured in LHC-8 medium containing L-glutamine, 10\% FBS, and $1 \%$ penicillin-streptomycin (Invitrogen).

Measurement of ROS production. Attached H292 cells were incubated by different concentrations of pyocyanin $(0-70 \mu \mathrm{M})$ for $2 \mathrm{~h}$. Intracellular superoxide production was measured by fluorescence of oxidized form of dihydroethidine ( $1 \mu \mathrm{M}$; Invitrogen; excitation: $485 \mathrm{~nm}$, emission: $590 \mathrm{~nm}$ ) using a fluorescent microscope (Leica, Wetzlar, Germany). Intracellular $\mathrm{H}_{2} \mathrm{O}_{2}$ production was followed by DCFDA (dichlorodihydrofluorescein diacetate) oxidation ( $20 \mu \mathrm{M}$; Invitrogen, excitation: $485 \mathrm{~nm}$, emission: $535 \mathrm{~nm}$ ) in a Fluoroskan plate reader (Thermo Scientific, Hudson, NH). Extracellular $\mathrm{H}_{2} \mathrm{O}_{2}$ was followed by measuring resorufin (excitation: $530 \mathrm{~nm}$, emission: $590 \mathrm{~nm}$ ) formation from Amplex Red (Invitrogen, Carlsbad, CA) by horseradish peroxidase $(20 \mathrm{U} / \mathrm{ml})$ in a Fluoroskan plate reader.

Sample preparation for microarrays. H292 cells were treated with or without $8 \mu \mathrm{M}$ pyocyanin for $48 \mathrm{~h}$ in serum-free RPMI medium. RNA was isolated as described (Supplementary Methods online) and an additional clean-up step was introduced using Qiagen (Valencia, CA) RNeasy RNA-isolation kit. The prepared RNAs were run on an RNA gel to ascertain the quality of the preparations. RNA was isolated in four independent experiments, collected, and subjected to microarray analysis simultaneously.

Statistical analysis of microarray data. Signal intensity estimates ("medianSignal") from Feature Extraction (Agilent Technologies, Santa Clara, CA) were converted to $\log _{2}$ scale. Two-channel data within each array was LOESS normalized (Supplementary Methods online, ref. 24) and median normalized between arrays. $\log _{2}$ ratios and $P$ values were generated for the contrast between pyocyanin-treated and untreated samples via a mixed-effects model analysis of variance with arrays as a random effect. The significance level was calculated by FDR (Supplementary Methods online, ref. 23) at 0.05 which equated to a raw $P$ value of $10^{-1.80}$. Thresholds for probes used in further analysis were selected at $\mid \log _{2}$ ratio $\mid>2$ (fourfold difference in gene expression) and a stringent $P$ value of $<10^{-3}$ (see Supplementary Figure S4 online). This set comprises 389 probes representing 302 unique transcripts and 268 unique Entrez gene identifiers. JMP-Genomics v4.1 software (SAS Institute, Cary, NC) was used for data processing and statistical analysis. The microarray data have been deposited in NCBI's Gene Expression Omnibus and are accessible through GEO Series accession number GSE22430 (http://www.ncbi.nlm.nih.gov/geo/query/ acc.cgi acc $=$ GSE22430).

Functional annotation cluster analysis. A total of 268 Entrez gene identifiers were submitted to the Functional Annotation Clustering Tool at the DAVID website (http://david.abcc.ncifcrf.gov:8080/) (Supplementary Methods online, ref. 25 and 26). In our analysis, DAVID served to initially assign functional annotation from the "GOTERM_BP_FAT" category to each gene, and to identify statistically significant associations to sets of genes both at the level of individual functional terms and for clusters of functions. The analysis was run under default options, and the resulting output was filtered for association of genes with GOTERM BP_FAT terms at a raw $P$ value $<0.01$. DAVID assigned 131 genes to 130 functional terms under these conditions. In order to organize and visualize clusters of related genes and functions, a binary matrix encoding the association of each gene with each GO FAT term was subjected to a two-way hierarchical clustering within JMP-software; this process simultaneously groups genes according to similarity of functional profiles and groups functions by similarity of gene membership (Figure 5).

Data analysis (other than microarray studies). Data are expressed as mean \pm s.e.m. Significance was assessed using Student's $t$-test when independent variables were studied; by analysis of variance and post hoc Dunnett's test when results of trends were compared. Significant changes were marked as ${ }^{\star}$ when $P<0.05$, ${ }^{\star *}$ when $P<0.01$, and ${ }^{* * *}$ when $P<0.001$.

SUPPLEMENTARY MATERIAL is linked to the online version of the paper at http://www.nature.com/mi

\section{ACKNOWLEDGMENTS}

This work was supported by the Intramural Research Program of the $\mathrm{NIH}$, National Institute of Allergy and Infectious Diseases.

\section{DISCLOSURE}

The authors declared no conflict of interest.

(c) 2011 Society for Mucosal Immunology

\section{REFERENCES}

1. Murphy, T.F. Pseudomonas aeruginosa in adults with chronic obstructive pulmonary disease. Curr. Opin. Pulm. Med. 15, 138-142 (2009).

2. Zoumot, Z. \& Wilson, R. Respiratory infection in noncystic fibrosis bronchiectasis. Curr. Opin. Infect. Dis. 23, 165-170.

3. Campodonico, V.L., Gadjeva, M., Paradis-Bleau, C., Uluer, A. \& Pier, G.B. Airway epithelial control of $P$ seudomonas aeruginosa infection in cystic fibrosis. Trends Mol. Med. 14, 120-133 (2008).

4. Mogayzel, P.J. Jr \& Flume, P.A. Update in cystic fibrosis 2009. Am. J. Respir. Crit. Care Med. 181, 539-544.

5. Lau, G.W., Hassett, D.J. \& Britigan, B.E. Modulation of lung epithelial functions by Pseudomonas aeruginosa. Trends Microbiol. 13, 389-397 (2005).

6. Lau, G.W., Hassett, D.J., Ran, H. \& Kong, F. The role of pyocyanin in Pseudomonas aeruginosa infection. Trends Mol. Med. 10, 599-606 (2004).

7. Mahajan-Miklos, S., Tan, M.W., Rahme, L.G. \& Ausubel, F.M. Molecular mechanisms of bacterial virulence elucidated using a Pseudomonas aeruginosa-Caenorhabditis elegans pathogenesis model. Cell 96, 47-56 (1999).

8. Rahme, L.G. et al. Plants and animals share functionally common bacterial virulence factors. Proc. Natl Acad. Sci. USA 97, 8815-8821 (2000).

9. Lau, G.W. et al. The Drosophila melanogaster toll pathway participates in resistance to infection by the gram-negative human pathogen Pseudomonas aeruginosa. Infect. Immun. 71, 4059-4066 (2003).

10. Lau, G.W., Ran, H., Kong, F., Hassett, D.J. \& Mavrodi, D. Pseudomonas aeruginosa pyocyanin is critical for lung infection in mice. Infect. Immun. 72, 4275-4278 (2004).

11. Caldwell, C.C. et al. Pseudomonas aeruginosa exotoxin pyocyanin causes cystic fibrosis airway pathogenesis. Am. J. Pathol. 175, 2473-2488 (2009).

12. Fothergill, J.L. et al. Widespread pyocyanin over-production among isolates of a cystic fibrosis epidemic strain. BMC Microbiol. 7, 45 (2007).

13. Wilson, R. et al. Measurement of Pseudomonas aeruginosa phenazine pigments in sputum and assessment of their contribution to sputum sol toxicity for respiratory epithelium. Infect. Immun. 56, 2515-2517 (1988).

14. Murray, T.S., Egan, M. \& Kazmierczak, B.I. Pseudomonas aeruginosa chronic colonization in cystic fibrosis patients. Curr. Opin. Pediatr. 19, 83-88 (2007).

15. Hassett, D.J. et al. Pseudomonas aeruginosa hypoxic or anaerobic biofilm infections within cystic fibrosis airways. Trends Microbiol. 17, 130-138 (2009).

16. Machen, T.E. Innate immune response in CF airway epithelia: hyperinflammatory? Am. J. Physiol. Cell Physiol. 291, C218-230 (2006).

17. Rada, B. \& Leto, T.L. Redox warfare between airway epithelial cells and Pseudomonas: dual oxidase vs. pyocyanin. Immunol. Res. 43, 198-209 (2009).

18. Rose, M.C. \& Voynow, J.A. Respiratory tract mucin genes and mucin glycoproteins in health and disease. Physiol. Rev. 86, 245-278 (2006). 
19. Thai, P., Loukoianov, A., Wachi, S. \& Wu, R. Regulation of airway mucin gene expression. Annu. Rev. Physiol. 70, 405-429 (2008).

20. Yan, F. et al. Reactive oxygen species regulate $P$ seudomonas aeruginosa lipopolysaccharide-induced MUC5AC mucin expression via PKC-NADPH oxidase-ROS-TGF-alpha signaling pathways in human airway epithelial cells. Biochem. Biophys. Res. Commun. 366, 513-519 (2008).

21. Kim, H.J. et al. The role of Nox4 in oxidative stress-induced MUC5AC overexpression in human airway epithelial cells. Am. J. Respir. Cell Mol. Biol. 39, 598-609 (2008).

22. Shao, M.X. \& Nadel, J.A. Dual oxidase 1-dependent MUC5AC mucin expression in cultured human airway epithelial cells. Proc. Natl Acad. Sci. USA 102, 767-772 (2005).

23. Tyner, J.W. et al. Blocking airway mucous cell metaplasia by inhibiting EGFR antiapoptosis and IL-13 transdifferentiation signals. J. Clin. Invest. 116, 309-321 (2006).

24. Takeyama, K. et al. Epidermal growth factor system regulates mucin production in airways. Proc. Natl Acad. Sci. USA 96, 3081-3086 (1999).

25. Casalino-Matsuda, S.M., Monzon, M.E. \& Forteza, R.M. Epidermal growth factor receptor activation by epidermal growth factor mediates oxidant-induced goblet cell metaplasia in human airway epithelium. Am. J. Respir. Cell Mol. Biol. 34, 581-591 (2006).

26. Burgel, P.R. \& Nadel, J.A. Epidermal growth factor receptor-mediated innate immune responses and their roles in airway diseases. Eur. Respir. J. 32, 1068-1081 (2008).

27. Higashiyama, S. et al. Membrane-anchored growth factors, the epidermal growth factor family: beyond receptor ligands. Cancer. Sci. 99, 214-220 (2008).

28. Shao, M.X., Ueki, I.F. \& Nadel, J.A. Tumor necrosis factor alphaconverting enzyme mediates MUC5AC mucin expression in cultured human airway epithelial cells. Proc. Natl Acad. Sci. USA 100, 11618-11623 (2003).

29. Denning, G.M. et al. Pseudomonas pyocyanin increases interleukin-8 expression by human airway epithelial cells. Infect. Immun. 66, 5777-5784 (1998).

30. O'Malley, Y.Q. et al. The Pseudomonas secretory product pyocyanin inhibits catalase activity in human lung epithelial cells. Am. J. Physiol. Lung. Cell Mol. Physiol. 285, L1077-1086 (2003).

31. Li, J.D. et al. Transcriptional activation of mucin by Pseudomonas aeruginosa lipopolysaccharide in the pathogenesis of cystic fibrosis lung disease. Proc. Natl Acad. Sci. USA 94, 967-972 (1997).

32. Dohrman, A. et al. Mucin gene (MUC 2 and MUC 5AC) upregulation by Gram-positive and Gram-negative bacteria. Biochim. Biophys. Acta. 1406, 251-259 (1998).

33. Takeyama, K. et al. Oxidative stress causes mucin synthesis via transactivation of epidermal growth factor receptor: role of neutrophils. J. Immunol. 164, 1546-1552 (2000).

34. Burgel, P.R. \& Nadel, J.A. Roles of epidermal growth factor receptor activation in epithelial cell repair and mucin production in airway epithelium. Thorax 59, 992-996 (2004).
35. Lemjabbar, H. \& Basbaum, C. Platelet-activating factor receptor and ADAM10 mediate responses to Staphylococcus aureus in epithelial cells. Nat. Med. 8, 41-46 (2002).

36. Perrais, M., Pigny, P., Copin, M.C., Aubert, J.P. \& VanSeuningen, I. Induction of MUC2 and MUC5AC mucins by factors of the epidermal growth factor (EGF) family is mediated by EGF receptor/Ras/Raf/ extracellular signal-regulated kinase cascade and Sp1. J. Biol. Chem. 277, 32258-32267 (2002).

37. Levine, S.J. et al. Tumor necrosis factor-alpha induces mucin hypersecretion and MUC-2 gene expression by human airway epithelial cells. Am. J. Respir. Cell Mol. Biol. 12, 196-204 (1995).

38. Kim, Y.D. et al. Interleukin-1 beta induces MUC2 gene expression and mucin secretion via activation of PKC-MEK/ERK, and PI3K in human airway epithelial cells. J. Korean Med. Sci. 17, 765-771 (2002).

39. Nichols, D., Chmiel, J. \& Berger, M. Chronic inflammation in the cystic fibrosis lung: alterations in inter- and intracellular signaling. Clin. Rev. Allergy Immunol. 34, 146-162 (2008).

40. Chen, J. et al. Dysfunction of Nrf-2 in CF epithelia leads to excess intracellular $\mathrm{H}_{2} \mathrm{O}_{2}$ and inflammatory cytokine production. PLoS One 3, e3367 (2008).

41. Saba, S., Soong, G., Greenberg, S. \& Prince, A. Bacterial stimulation of epithelial G-CSF and GM-CSF expression promotes PMN survival in CF airways. Am. J. Respir. Cell Mol. Biol. 27, 561-567 (2002).

42. Gregory, A.D., Hogue, L.A., Ferkol, T.W. \& Link, D.C. Regulation of systemic and local neutrophil responses by G-CSF during pulmonary Pseudomonas aeruginosa infection. Blood 109, 3235-3243 (2007).

43. Stanley, E. et al. Granulocyte/macrophage colony-stimulating factordeficient mice show no major perturbation of hematopoiesis but develop a characteristic pulmonary pathology. Proc. Natl Acad. Sci. USA 91, 5592-5596 (1994).

44. Jame, A.J. et al. Human bronchial epithelial cells express an active and inducible biosynthetic pathway for leukotrienes B4 and C4. Clin. Exp. Allergy 37, 880-892 (2007).

45. Velazquez, J.R. \& Teran, L.M. Chemokines and their receptors in the allergic airway inflammatory process. Clin. Rev. Allergy Immunol. doi:10.1007/s12016-010-8202-6.

46. Sha, Q., Truong-Tran, A.Q., Plitt, J.R., Beck, L.A. \& Schleimer, R.P. Activation of airway epithelial cells by toll-like receptor agonists. Am. J. Respir. Cell Mol. Biol. 31, 358-364 (2004).

47. Dubin, P.J. \& Kolls, J.K. IL-23 mediates inflammatory responses to mucoid Pseudomonas aeruginosa lung infection in mice. Am. J. Physiol. Lung Cell Mol. Physiol. 292, L519-528 (2007).

48. Bruscia, E. et al. Isolation of CF cell lines corrected at DeltaF508CFTR locus by SFHR-mediated targeting. Gene Ther. 9, 683-685 (2002).

49. Rada, B., Lekstrom, K., Damian, S., Dupuy, C. \& Leto, T.L. The Pseudomonas toxin pyocyanin inhibits the dual oxidase-based antimicrobial system as it imposes oxidative stress on airway epithelial cells. J. Immunol. 181, 4883-4893 (2008). 